\title{
A COSSERAT-TYPE PLATE THEORY AND ITS APPLICATION TO CARBON NANOTUBE MICROSTRUCTURE
}

\author{
${ }^{1}$ Abdellatif Selmi, ${ }^{1}$ Hedi Hassis, ${ }^{2}$ Issam Doghri and ${ }^{1}$ Hatem Zenzri \\ ${ }^{1}$ Civil Engineering Laboratory, Ecole Nationale d'Ingénieurs de Tunis, B.P.37, Le belvédère 1002, Tunis, Tunisia \\ ${ }^{2}$ CESAME, UCL, Louvain-la-Neuve, Belgium
}

Received 2013-04-12; Revised 2013-07-16; Accepted 2014-05-15

\begin{abstract}
The predictive capabilities of plate and shell theories greatly depend on their underlying kinematic assumptions. In this study, we develop a Cosserat-type elastic plate theory which accounts for rotations around the normal to the mid-surface plane (so-called drilling rotations). Internal loads, equilibrium equations, boundary conditions and constitutive equations are derived. The case of a Single Walled carbon Nanotube (SWNT) modelled as a Cosserat medium is taken here as a reference example. Material parameters are identified and the proposed theory is used to solve analytically the problem of a polymer-SWNT composite tube under torsion. Predictions such as an absolute size effect are compared to those of the classical Cauchy-de Saint-Venant results.
\end{abstract}

Keywords: Plate Theory, Drilling Rotation, Cosserat Medium, Carbon Nanotube, Composite Microstructure

\section{INTRODUCTION}

SWNT are graphene cylinders that typically have diameters of about $1 \mathrm{~nm}$ with lengths of several microns (Selmi et al., 2007). Due to their extraordinary mechanical properties, SWNT have become objects of intensive investigation for different applications (Varthamanan, 2013). Because of the high aspect ratio (thousands) in addition to the mechanical strength, they have become very promising components for composites (Meoli and Plumlee, 2002; Partridge et al., 2000). To improve the performance of a matrix or to achieve new properties, extensive studies have been devoted to the use of SWNT as reinforcing material. Indeed, there are researchers who have demonstrated improvement in properties of pitch and polystyrene (Quin et al., 2000) and poly (p-phenylene benzobisoxazole) (Kumar et al., 2002).

In the mechanical study of SWNT material, computational chemistry models predict molecular properties based on known quantum interactions and computational solid mechanics models predict the macroscopic mechanical behaviour of materials idealized as continuous media based on known bulk material properties (Odegard et al., 2003).

However, a corresponding model does not exist in the intermediate length scale range. If a hierarchical approach is used to model the macroscopic behavior of nano-structured materials, then a methodology must be developed to link the molecular structure and the macroscopic properties. An important component in molecular dynamics calculations of the nano-structure of a material is the description of the forces between individual atoms and the energies associated with bond stretching, angle variation, torsion and inversion.

In order to simplify the calculation of the total molecular potential energy of molecular models with complex molecular structures and loading conditions, an intermediate model may be used to substitute the molecular model (Odegard et al., 2002; 2003).

The mechanical representation of the lattice behaviour serves as an intermediate step in linking the molecular potential with an equivalent-continuum model. In the truss model, each truss element corresponds to a chemical bond or a significant non-

Corresponding Author: Abdellatif Selmi, Civil Engineering Laboratory, Ecole Nationale d'Ingénieurs de Tunis, B.P.37, Le belvédère 1002, Tunis, Tunisia 
bonded interaction. The stretching potential of each bond corresponds to the stretching of the corresponding truss element; see Fig. 1.

In previous studies, the angle variation (associated to a drilling moment) phenomenon is related to in-plane forces giving the same energy associated with the angle variation; see Fig. 1.

In the present work, the angle variation is considered by adding a new Degree Of Freedom (DOF): The drilling rotation. Then a drilling moment in the microstructure of the nanotube is introduced making the classical plate theory invalid. The theory presented in sections 2 and 3 will be applied to the nanotube microstructure.

Shell and plate theories are the subject of a very large body of literature. These theories are greatly dependent on the models adopted to represent shell structures and the processes of their deformation. They differ with respect to the consideration of a linear or non-linear model, to the variations of the in-plane displacement components across the thickness and to the kinematic description (Rubin, 2000). Most of classical plate theories involve: An in-plane normal stress tensor, N, an in-plane moment tensor, $\left(\mathrm{M}_{11}\right)$ and $\left(\mathrm{M}_{22}\right)$ are bending moments, while $\mathrm{M}_{12}$ and $\mathrm{M}_{21}$ are twisting moments (all are per unit length). In this study, we will designate them collectively as "in-plane moments") and a shear stress vector, $\vec{T}$. The kinematics is defined by the displacement of the middle plane, $\vec{U}$ and the in-plane rotation of the normal fiber, $\vec{\Omega}_{\omega}$ (Fig. 2).

Classical shell and plate theories based on the LoveKirchhoff assumption and the Green strain measure are fully developed by (Pietraszkiewicz, 1984). These two-dimensional theories are, in fact, 2D approximations to $3 \mathrm{D}$ elastic models based on a priori assumptions regarding the variation of the unknowns (i.e., the displacements and the stresses) across the thickness of the plate.

A method called asymptotic expansion has been used to obtain two-dimensional models of thin elastic plates. In that method, a power series expansion of the three-dimensional solution is used by considering the thickness of the plate as the small parameter. The asymptotic approach has also been applied to derive the fully non-linear model of thin plates that are made of a general non-linear elastic material. In the study of Erbay (1997), there is an abstract of this study along with an asymptotic membrane theory of thin hyperelastic plates.
Based on the Love-Kirchhoff model, the displacement components through the thickness were estimated to be linear or higher order series expansion (Kreja and Schmidt, 2006). Classical theories are unable to explain some phenomena at high frequencies and small wavelengths of surface waves, in addition they are insufficient in the elucidation of granular and large molecular materials such as polymers (Kreja and Schmidt, 2006). Such limitations are due to the fact that the interaction cannot be described only by a force vector; a couple or moment vector is also needed. This interpretation led investigators to enrich the kinematical description and various theories have been proposed in recent years with various kinematic variables.

The construction of a plate theory based on the linear theory of micropolar elasticity is not a new subject and it attracts considerable interest from researchers (Ambartsumian, 2002). To derive his micropolar plate theory, (Eringen, 1967) assumed that the stress and displacement fields do not vary rapidly across the thickness of the plate. The three-dimensional field equations of micropolar elasticity are integrated across the thickness and the balance equations of micropolar elastic plates are derived. Next, assumptions regarding the variation of the unknowns across the thickness of the plate are made.

The asymptotic expansion technique was extended to obtain the two-dimensional dynamic equations of thin micropolar elastic plates from the threedimensional dynamic equations of micropolar elasticity theory (Erbay, 2000). It consists in scaling the field variables by an appropriate thickness parameter to reflect the expected behaviour of the plate. A power series expansion of the threedimensional solution is used by considering the thickness parameter as a small parameter.

In the finite strain case, based on the polar decomposition theorem and the Love-Kirchhoff assumption, Atluri (1984) has developed a shell theory which includes a rotation tensor related the definition of the stress measure. Similarly, (Sansour and Bocko, 1998) have developed a non-linear theory with exact description of the kinematic field, in which the rotation tensor describes the rotations DOF of any material segment and takes into account the drilling rotations in a natural way.

In the classical plate theories, the drilling rotation (i.e., rotation around the normal to the mid-surface plane) and the drilling moment are nil (Reissner, 1985). 


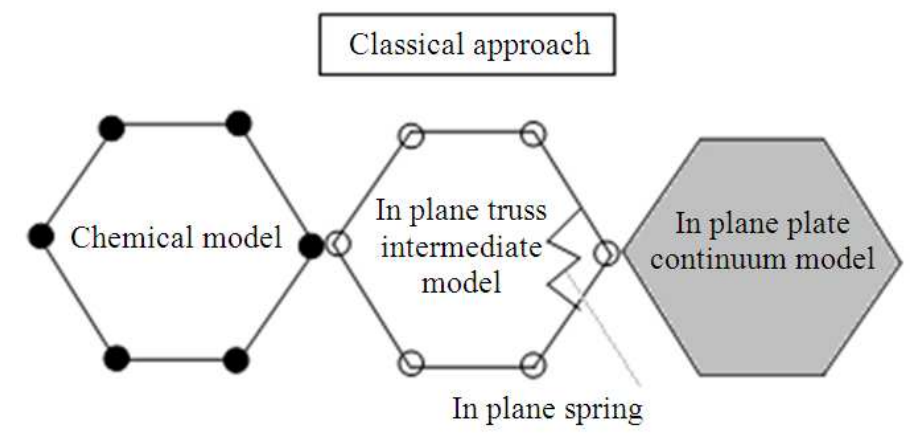

Fig. 1. Classical model for nanotube microstructure

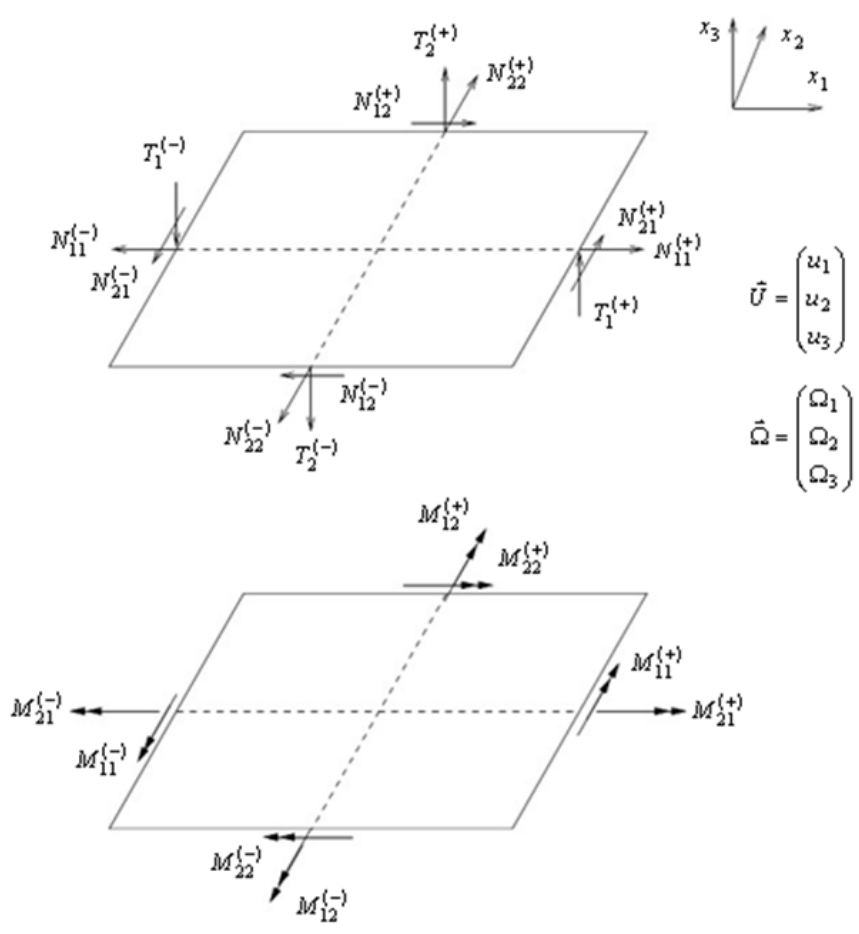

Fig. 2. Classical internal loads and generalized displacement

In some applications, such as honey-comb structures and Carbon nanotube microstructures, an in-plane extension induces a drilling rotation and out-of-plane internal loads (moment and shear). Compared to classical theories of plates and shells, this coupling between extension and drilling rotation induces a non-symmetric normal stress tensor and new internal loads. A plate with a drilling rotation will be called "an out-of-plane Cosserat plate".

Existing theories which deal with drilling rotation are geometrically non-linear. However, in order to clarify the behaviour of granular and large molecular materials such as polymers in which the transmission of interaction may be not only through a force vector but also through a couple or moment vector and in which size effects could be important, considering an additional DOF like drilling rotation in the linear elastic domain has a great interest.

In this study, using the virtual principle work, an elastic out-of-plane Cosserat plate theory is developed. The internal loads, the equilibrium equations, the boundary conditions and the elastic constitutive equations are derived. 
The case of nanotube microstructures will be the reference application. An illustration of the drilling rotations effect will be presented in the examples of the torsion of SWNT and the torsion of SWNT/Polymer composite beams.

\section{EQUILIBRIUM EQUATIONS AND BEHAVIOUR OF A CONTINUUM PLATE WITH COSSERAT DRILLING EFFECT}

Let us consider a plate defined geometrically by a middle plane $\omega$ in which the position of a point is defined by the coordinates $\left(\mathrm{x}_{1}, \mathrm{x}_{2}\right)$. For any material segment initially normal to $\omega$, the kinematics is characterized by a displacement field $\vec{U}$ and a rotation $\vec{\Omega}$ defined by Equation 1:

$$
\begin{aligned}
& \vec{U}=\left(\begin{array}{l}
u_{1}\left(x_{1}, x_{2}\right) \\
u_{2}\left(x_{1}, x_{2}\right) \\
u_{3}\left(x_{1}, x_{2}\right)
\end{array}\right)=\left(\begin{array}{l}
u_{1} \\
u_{2} \\
u_{3}
\end{array}\right)=\left(\begin{array}{l}
\vec{u}_{\omega} \\
u_{3}
\end{array}\right) \\
& \vec{\Omega}=\left(\begin{array}{l}
\Omega_{1}\left(x_{1}, x_{2}\right) \\
\Omega_{2}\left(x_{1}, x_{2}\right) \\
\Omega_{3}\left(x_{1}, x_{2}\right)
\end{array}\right)=\left(\begin{array}{l}
\Omega_{1} \\
\Omega_{2} \\
\Omega_{3}
\end{array}\right)=\left(\begin{array}{c}
\vec{\Omega}_{\omega} \\
\Omega_{3}
\end{array}\right)
\end{aligned}
$$

The classical plate models consider that the plate is a two-dimensional in-plane Cauchy medium $\left(\Omega_{3}=0\right)$. The model considered here takes into account the drilling rotation $\Omega_{3}$; the plate is then a two dimensional in- and out-of-plane Cosserat medium.

The virtual work principle is used hereafter to derive the equilibrium equations and the boundary conditions of the continuum plate with a drilling Cosserat effect.

\subsection{Virtual Work of Internal Loads}

In the following and as classically considered, the virtual work is an integration of a volumetric density over the system or any subsystem.

The internal virtual work is chosen as a linear functional of the virtual kinematic variables and their first gradients:

$$
P_{i}=-\int_{\omega}\left(\vec{A} \cdot \vec{U}+{ }^{t} \widehat{N}: \nabla \vec{U}+\vec{B} \cdot \vec{\Omega}+{ }^{t} \hat{M}: \nabla \vec{\Omega}\right) d \omega
$$

where, $\vec{A}, \vec{B}, \hat{N}$ and $\hat{M}$ are internal efforts associated to the kinematic variables, introduced systematically and will be discussed later.
The kinematic variable gradients $(\nabla \vec{U}, \nabla \vec{\Omega})$ and the internal efforts $(\hat{N}, \hat{M}, \vec{B})$ are divided into in- and out-ofplane components which are written in matrix form in an orthonormal basis as follows. Equation 3 to 7 :

$$
\begin{aligned}
& \nabla \vec{U}=\left[\begin{array}{lll}
u_{1,1} & u_{1,2} & 0 \\
u_{2,1} & u_{2,2} & 0 \\
u_{3,1} & u_{3,2} & 0
\end{array}\right]=\left[\begin{array}{cc}
\nabla_{\omega} \vec{u}_{\omega} & \overrightarrow{0}_{\omega} \\
{ }^{t} \vec{\nabla}_{\omega} u_{3} & 0
\end{array}\right] \\
& \nabla \vec{\Omega}=\left[\begin{array}{lll}
\Omega_{1,1} & \Omega_{1,2} & 0 \\
\Omega_{2,1} & \Omega_{2,2} & 0 \\
\Omega_{2,1} & \Omega_{3,2} & 0
\end{array}\right]=\left[\begin{array}{cc}
\nabla_{\omega} \vec{\Omega}_{\omega} & \overrightarrow{0}_{\omega} \\
{ }^{t} \vec{\nabla}_{\omega} \Omega_{3} & 0
\end{array}\right] \\
& \hat{N}=\left[\begin{array}{ccc}
N_{11} & N_{12} & 0 \\
N_{21} & N_{22} & 0 \\
T_{1} & T_{2} & 0
\end{array}\right]=\left[\begin{array}{cc}
N & \overrightarrow{0}_{\omega} \\
{ }^{t} \vec{T} & 0
\end{array}\right] \\
& \hat{M}=\left[\begin{array}{ccc}
M_{11} & M_{12} & 0 \\
M_{21} & M_{22} & 0 \\
C_{1} & C_{2} & 0
\end{array}\right]=\left[\begin{array}{cc}
\vec{M} & \overrightarrow{0}_{\omega} \\
{ }^{t} \vec{C} & 0
\end{array}\right] \\
& \vec{B}=\left(\begin{array}{c}
B_{1} \\
B_{2} \\
B_{3}
\end{array}\right)=\left(\begin{array}{c}
\vec{B} \omega \\
D
\end{array}\right)
\end{aligned}
$$

With decomposition to in-plane and out-of-plane parts, the internal virtual work takes the following expression Equation 8:

$$
\begin{aligned}
& P_{i}=-\int_{\omega}\left(\vec{A} \cdot \vec{U}+{ }^{t} N: \nabla_{\omega} \vec{u}_{\omega}+\vec{T} \cdot \vec{\nabla}_{\omega} u_{3}+\vec{B}_{\omega} \cdot \vec{\Omega}_{\omega}\right) d \omega \\
& -\int_{\omega}\left(D \Omega_{3}+{ }^{t} \bar{M}: \nabla_{\omega} \vec{\Omega}_{\omega}+\vec{C} \cdot \vec{\nabla}_{\omega} \Omega_{3}\right) d \omega
\end{aligned}
$$

According to the first statement of the virtual work principle, the virtual work of internal loads associated to any virtual rigid body motion is equal to zero. This leads to:

For a translation motion defined by: $\vec{U}=\vec{U}^{0}$, one obtains $(\vec{U}, \vec{\Omega})=\left(\vec{U}^{0}, \overrightarrow{0}\right)$. Then Equation 9 and 10 :

$$
P_{i}=-\int_{\omega}\left(\vec{A} \cdot \vec{U}^{0} d \omega=0, \quad \forall \vec{U}^{0} \quad \Rightarrow \quad \vec{A}=0\right.
$$

For a rigid rotation defined by: $\vec{U}=\vec{\Omega}^{0} \wedge \vec{x}$, where $\vec{x}$ is the position vector and $\vec{\Omega}^{0}$ is a constant vector, one obtains $(\vec{U}, \vec{\Omega})=\left(\vec{\Omega}^{0} \wedge \vec{x}, \vec{\Omega}^{0}\right)$.

Where: 


$$
\vec{\Omega}^{0}=\left(\begin{array}{c}
\Omega_{1}^{0} \\
\Omega_{2}^{0} \\
\Omega_{3}^{0}
\end{array}\right)=\left(\begin{array}{c}
\vec{\Omega}_{\omega}^{0} \\
\Omega_{3}^{0}
\end{array}\right)
$$

Using the classical plate's rotations defined by Equation 11:

$$
\vec{\beta}_{\omega}^{0}=\left(\begin{array}{c}
\Omega_{2}^{0} \\
-\Omega_{1}^{0}
\end{array}\right)
$$

The internal virtual work is reduced to Equation 12:

$$
\begin{aligned}
& P_{i}=-\int_{\omega}\left[\left(N_{21}-N_{12}+D\right) \cdot \Omega_{3}^{0}-\vec{T} \cdot \vec{\beta}_{\omega}^{0}+\vec{B}_{\omega} \cdot \vec{\Omega}_{\omega}^{0}\right] d \omega=0 \\
& , \forall \vec{\Omega}^{0}
\end{aligned}
$$

And leads to Equation 13:

$$
D=N_{12}-N_{21} \text { and } \vec{T}={ }^{t} R \vec{B}_{\omega}
$$

Where: $\quad R=\left[\begin{array}{cc}0 & -1 \\ 1 & 0\end{array}\right]$

Finally, for any virtual motion the internal virtual work takes the following expression:

$$
\begin{aligned}
& P_{i}=-\int_{\omega}\left({ }^{t} N: \nabla_{\omega} \vec{u}_{\omega}+\vec{T} \cdot \vec{\nabla}_{\omega} u_{3}+\vec{T} \cdot \vec{\beta}_{\omega}\right) d \omega \\
& -\int_{\omega}\left(D \beta_{3}+{ }^{t} M: \nabla_{\omega} \vec{\beta}_{\omega}+\vec{C} \cdot \vec{\nabla}_{\omega} \beta_{3}\right) d \omega
\end{aligned}
$$

Where:

$$
M={ }^{t} R \cdot \bar{M}, \quad \beta_{3}=\Omega_{3} \quad \text { and } \quad \vec{\beta}_{\omega}=R^{-1} \cdot \vec{\Omega}_{\omega}
$$

Using Green's integration, the internal virtual work takes the following expression:

$$
\begin{aligned}
& P_{i}=\int_{\omega}\left(\operatorname{div} N \cdot \vec{u}_{\omega}+\operatorname{div} \vec{T} \cdot u_{3}-\vec{T} \cdot \vec{\beta}_{\omega}-D \beta_{3}+\operatorname{div} M \cdot \vec{\beta}_{\omega}\right. \\
& \left.+\operatorname{div} \vec{C} \cdot \beta_{3}\right) d \omega-\int_{\partial \omega}\left(N \cdot \vec{v} \cdot \vec{u}_{\omega}+\vec{T} \cdot \vec{v} \cdot u_{3}+M \cdot \vec{v} \cdot \vec{\beta}_{\omega}+\vec{C} \cdot \vec{v} \beta_{3}\right) d \Gamma
\end{aligned}
$$

where, $\partial \omega$ is the boundary of $\omega$ and $\vec{v}$ is the unit normal vector of $\partial \omega$ and $\vec{v}$ situated in the mid-plane.

\subsection{Virtual Work of External Loads}

The external virtual work is written as a linear form of the kinematical variables. Equation 17:

$$
\begin{aligned}
& P_{e}=\int_{\omega}\left(\vec{F}_{\omega} \cdot \vec{u}_{\omega}+F_{3} u_{3}+\vec{m}_{\omega} \cdot \vec{\beta}_{\omega}+m_{3} \beta_{3}\right) d \omega \\
& +\int_{\mathbb{T} \omega}\left(\vec{F}_{s \omega} \cdot \vec{u}_{\omega}+F_{s 3} u_{3}+\vec{m}_{s \omega} \cdot \vec{\beta}_{\omega}+m_{s 3} \beta_{3}\right) d
\end{aligned}
$$

where, the external loads associated respectively with the middle surface and its boundary are the following:

$\vec{F}_{\omega}$ and $\vec{F}_{s \omega}:$ In-plane density forces

$\mathrm{F}_{3}$ and $\mathrm{F}_{\mathrm{s} 3}$ : Out-of-plane density forces

$\vec{m}_{\omega}$ and $\vec{m}_{s \omega}$ : In plane density moments

$\mathrm{m}_{3}$ and $\mathrm{m}_{\mathrm{s} 3}$ : Density moments of axis the normal $\overrightarrow{\mathrm{n}}$ to the mid-surface plane

\subsection{Derivation of Equilibrium Equations and Boundary Conditions}

Assuming the continuity of the function fields $(\vec{U}, \vec{\Omega})$, using the divergence theorem and the second postulate of the virtual principle work, which is:

$$
P_{i}+P_{e}=0
$$

Equation 18 leads to the following equilibrium:

$$
\left\{\begin{array}{l}
\operatorname{div} N+\vec{F}_{\omega}=0 \\
\operatorname{div} \vec{T}+F_{3}=0 \\
\operatorname{div} M-\vec{T}+\vec{m}_{\omega}=0 \\
\operatorname{div} \vec{C}-D+m_{3}
\end{array}\right.
$$

And the following boundary conditions:

$$
\left\{\begin{array}{l}
-N \cdot \vec{v}+\vec{F}_{s \omega}=0 \\
-\vec{T} \cdot \vec{v}+F_{s 3}=0 \\
-M \cdot \vec{v}+\vec{m}_{s \omega}=0 \\
-\vec{C} \cdot \vec{v}+m_{s 3}=0
\end{array}\right.
$$

Equation 20 gives an interpretation of the internal loads introduced in Equation 2 and Fig. 3 describes them.

Let us consider an elementary plane surface whose facets have an outer unit normal $\vec{v}$ equal to $\pm \vec{x}_{1}$ or $\pm \vec{x}_{2}$. From Equation 20, we interpret that:

$$
\begin{aligned}
& N \cdot \vec{v}=\text { The in-plane force } \\
& M \cdot \vec{v}=\text { The in-plane moment } \\
& \vec{T} \cdot \vec{v}=\text { The shear force } \\
& \vec{C} \cdot \vec{v}=\text { The drilling moment }
\end{aligned}
$$



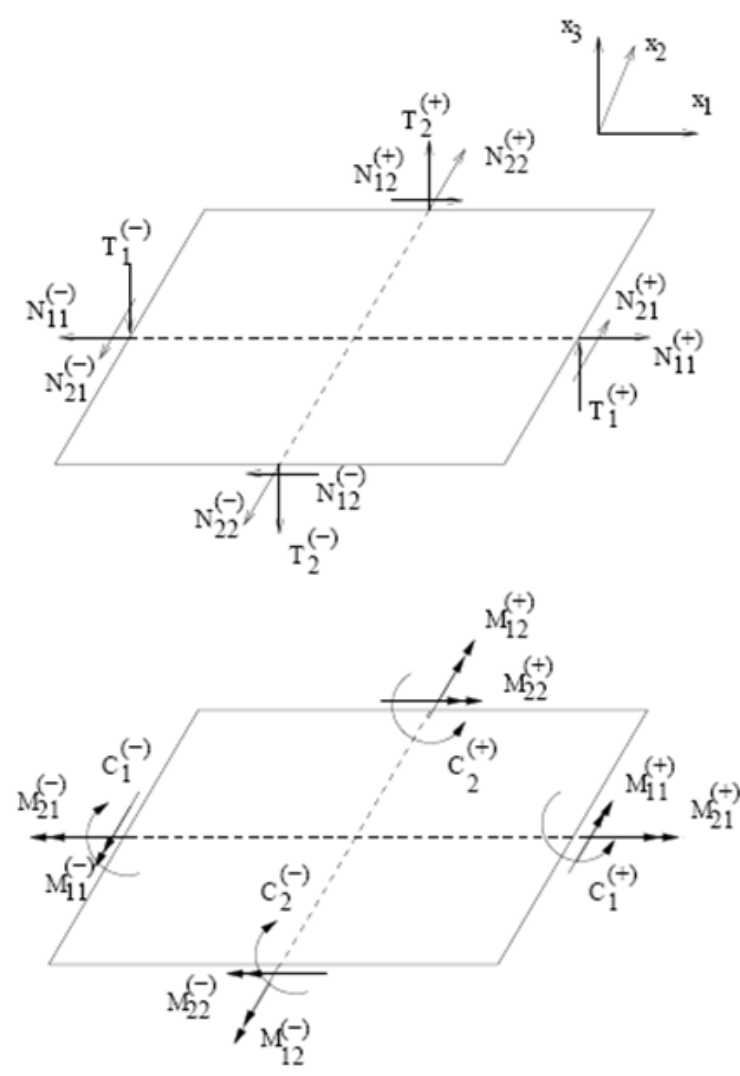

Fig. 3. Description of the internal loads in the proposed Cosserat-type theory

\subsection{Elastic Constitutive Behavior}

Using the following state variables:

$$
\left\{\begin{array}{l}
\varepsilon_{\omega}=\frac{1}{2}\left(\nabla_{\omega} \vec{u}_{\omega}+{ }^{t} \nabla_{\omega} \vec{u}_{\omega}\right) \\
\left.\vec{\theta}=\vec{\nabla}_{\omega} u_{3}+\vec{\beta}_{\omega}\right) \\
\lambda_{\omega}=\frac{1}{2}\left(\nabla_{\omega} \vec{\beta}_{\omega}+{ }^{t} \nabla_{\omega} \vec{\beta}_{\omega}\right) \\
\vec{\lambda}_{3}=\vec{\nabla}_{\omega} \beta_{3} \\
\beta_{3}
\end{array}\right.
$$

Which are respectively the in-plane infinitesimal symmetric strain tensor, the total in-plane rotation, the in-plane infinitesimal symmetric rotation gradient tensor and the drilling rotation gradient. The deformation power density is Equation 22:

$$
P_{d e f}=N^{s}: \dot{\varepsilon}_{\omega}+\vec{T} \cdot \dot{\vec{\theta}}+M: \dot{\lambda}_{\omega}+\vec{C} \cdot \dot{\vec{\lambda}}_{3}+D \dot{\beta}_{3}
$$

where, $\mathrm{N}^{\mathrm{s}}$ stands for the symmetric part of the normal tensor $\mathrm{N}$.

The Clausius-Duhem inequality implies that for any evolution the dissipative power density has to be positive, that is Equation 23:

$$
\begin{aligned}
& \forall \quad\left(\dot{\varepsilon}_{\omega}, \dot{\vec{\theta}}, \dot{\lambda}_{\omega}, \dot{\vec{\lambda}}_{3}, \dot{\beta}_{3}\right), \\
& \left(N^{s}-\frac{\partial \psi}{\partial \varepsilon_{\omega}}\right): \dot{\varepsilon}_{\omega}+\left(\vec{T}-\frac{\partial \psi}{\partial \vec{\theta}}\right) \cdot \dot{\vec{\theta}}+\left(M-\frac{\partial \psi}{\partial \lambda_{\omega}}\right): \dot{\lambda}_{\omega} \\
& +\left(\vec{C}-\frac{\partial \psi}{\partial \vec{\lambda}_{3}}\right) \cdot \dot{\vec{\lambda}}_{3}+\left(D-\frac{\partial \psi}{\partial \beta_{3}}\right) \dot{\beta}_{3} \geq 0
\end{aligned}
$$

where, $\psi$ is the thermodynamic potential. An elastic and linear behaviour is then chosen as follows:

$$
\begin{aligned}
& \psi=\frac{1}{2} \varepsilon_{\omega}: C_{N}: \varepsilon_{\omega}+\frac{1}{2} \vec{\theta} L \cdot \vec{\theta}+\beta_{3} \delta \beta_{3} \\
& +\frac{1}{2} \lambda_{\omega}: C_{M}: \lambda_{\omega}+\frac{1}{2} \vec{\lambda}_{3} v \cdot \vec{\lambda}_{3}+\varepsilon_{\omega}: K \cdot \vec{\lambda}_{3}
\end{aligned}
$$

where, $C_{N}$ and $C_{M}$ are fourth order tensor, $K$ is a third order tensor, $L, \delta$ and $v$ are real constants.

This choice includes a coupling between $\varepsilon_{\omega}$ and $\lambda_{3}$, this allows to take into account the coupling between inplane extension and drilling rotation.

For a reversible process, the dissipative power density is nil. Thus, the behaviour relations are:

$$
\left\{\begin{array}{l}
N^{s}=\frac{\partial \psi}{\partial \varepsilon_{\omega}}=C_{N}: \varepsilon_{\omega}+K \cdot \vec{\lambda}_{3} \\
\vec{T}=\frac{\partial \psi}{\partial \vec{\theta}}=L \cdot \vec{\theta} \\
M=\frac{\partial \psi}{\partial \lambda_{\omega}}=C_{M}: \lambda_{\omega} \\
\vec{C}=\frac{\partial \psi}{\partial \vec{\lambda}_{3}}=v \cdot \vec{\lambda}_{3}+\varepsilon_{\omega}: K \\
D=\frac{\partial \psi}{\partial \beta_{3}}=N_{12}-N_{21}=2 \delta \beta_{3}
\end{array}\right.
$$

In the case of 2D linear isotropic elastic theory, the third order tensor $K$ is nil, so the behaviour laws are written as:

$$
\left\{\begin{array}{l}
N^{s}=\alpha \varepsilon_{\omega}+\eta\left(\operatorname{tr\varepsilon } \varepsilon_{\omega}\right) I \\
\vec{T}=L \cdot \vec{\theta} \\
M=\varphi \lambda_{\omega}+\varphi\left(t r \lambda_{\omega}\right) I \\
\vec{C}=v \cdot \vec{\lambda}_{3} \\
D=N_{12}-N_{21}=2 \delta \beta_{3}
\end{array}\right.
$$


The following decomposition is introduced:

$$
\begin{aligned}
& N=N^{s}+N^{s s}=\left[\begin{array}{cc}
N_{11} & \frac{N_{12}+N_{21}}{2} \\
\frac{N_{21}+N_{12}}{2} & N_{22}
\end{array}\right] \\
& +\left[\begin{array}{cc}
0 & \frac{N_{12}-N_{21}}{2} \\
\frac{N_{21}-N_{12}}{2} & 0
\end{array}\right] \\
& =N^{s}+\beta_{3}\left[\begin{array}{ll}
0 & \delta \\
-\delta & 0
\end{array}\right] \\
& =N^{s}+\beta_{3} \Delta
\end{aligned}
$$

where, $\mathrm{N}^{\mathrm{ss}}$ stands for the skew-symmetric part of the normal tensor $\mathrm{N}$, "I" is the second-order identity tensor and $(\alpha, \eta, \delta, L, \varphi, \varphi, v)$ are mechanical characteristics which will be identified later.

Finally, the first constitutive equation in Equation 26 is replaced as follows:

$$
N=\alpha \varepsilon_{\omega}+\eta\left(\operatorname{tr} \varepsilon_{\omega}\right) I+\beta_{3} \Delta
$$

\section{Remark}

Compared to the literature where the normal tensor $\mathrm{N}$ is symmetric, from the behaviour laws derived for the developed theory, $\mathrm{N}$ (Equation 28) is not symmetric. We remark also the existence of two additional constitutive Equation 26d and 26e which are obviously satisfied when the drilling rotation $\beta_{3}$ is nil.

If we do not take the drilling rotation $\beta_{3}$ into account, $\mathrm{N}$ will be symmetric see Equation 28 ) then $\mathrm{D}$ and $\overrightarrow{\mathrm{C}}$ will be nil (see Equation 26e, 21e and 26d). Therefore we retrieve the constitutive equations, the equilibrium equations and the boundary conditions given by classical theories of Reissner (1985).

\section{APPLICATION: CARBON NANOTUBE MICROSTRUCTURE}

\subsection{Introduction}

Nano-structured materials have generated considerable interest in the materials research community in the last few years partly due to their potentially remarkable mechanical properties. This is particularly true for Carbon nanotubes which are sheets of graphene which are rolled up and can be capped at their ends (Selmi et al., 2007).

In micromechanical analyses, the method of unit cells has been used to determine the effective properties of heterogeneous materials by identifying and analysing convenient domains of repeating microstructure. In the current study, a repeating unit of nanotubes is defined as a truss microstructure and homogenized to a two dimensional continuum plate, as described in Fig. 4.

To reduce the computational time associated with modelling the graphene sheet, a Representative Volume Element (RVE) for graphene is used in this study (Fig. 5). The selected RVE allows each DOF of the Carbon atom associated with bond stretching and bond-angle variation in the hexagonal ring to be completely modelled by truss and continuum finite element model nodal-displacement degrees of freedom. Also, this RVE allows the displacements on the boundary of the proposed chemical, truss and continuum models to coincide exactly. Furthermore, macroscopic loading conditions applied to a continuous graphene plate can be easily reduced to periodic boundary conditions that are applied to the RVE.

The elastic constants associated with the truss structure (Fig. 6) are determined using a computational program (SAP, 2000) with the following data: Distance between two successive atoms is constant and equal to $\mathrm{R}^{\mathrm{a}}=0.14 \mathrm{~nm}$, the stiffness associated with rotational spring is $K^{\theta}=8.76 \times 10^{-10} \frac{n J}{\text { angle.rad }^{2}}$ and the rods are characterized by a Young modulus equal to $1.005 \mathrm{TPa}$ and a diameter equal to $0.34 \mathrm{~nm}$ (Odegard et al., 2002; Natsuki et al., 2004).

\subsection{Results}

Using models described by Fig. 6, the constitutive parameters associated with the continuum model are identified. For example, for the determination of a and $\delta$, Fig. 6b and c, from the constitutive Equation 28 and 29, we have:

$$
\left\{\begin{array}{l}
N_{12}=\frac{\alpha}{2}\left(u_{1,2}+u_{2,1}\right)+\delta \beta_{3} \\
N_{2 l}=\frac{\alpha}{2}\left(u_{2, I}+u_{l, 2}\right)-\delta \beta
\end{array}\right.
$$




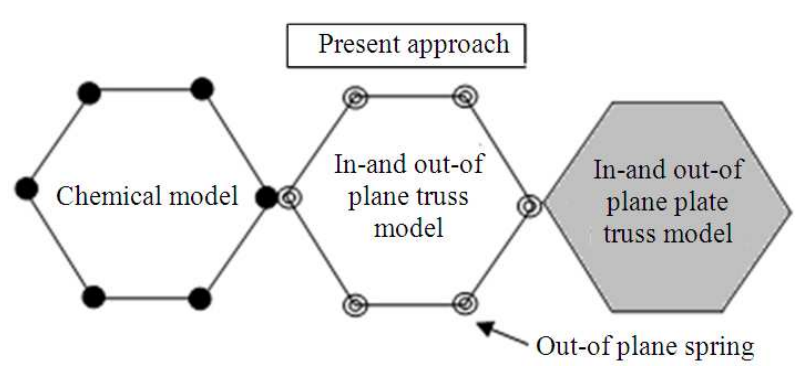

Fig. 4. Present model for nanotube microstructure
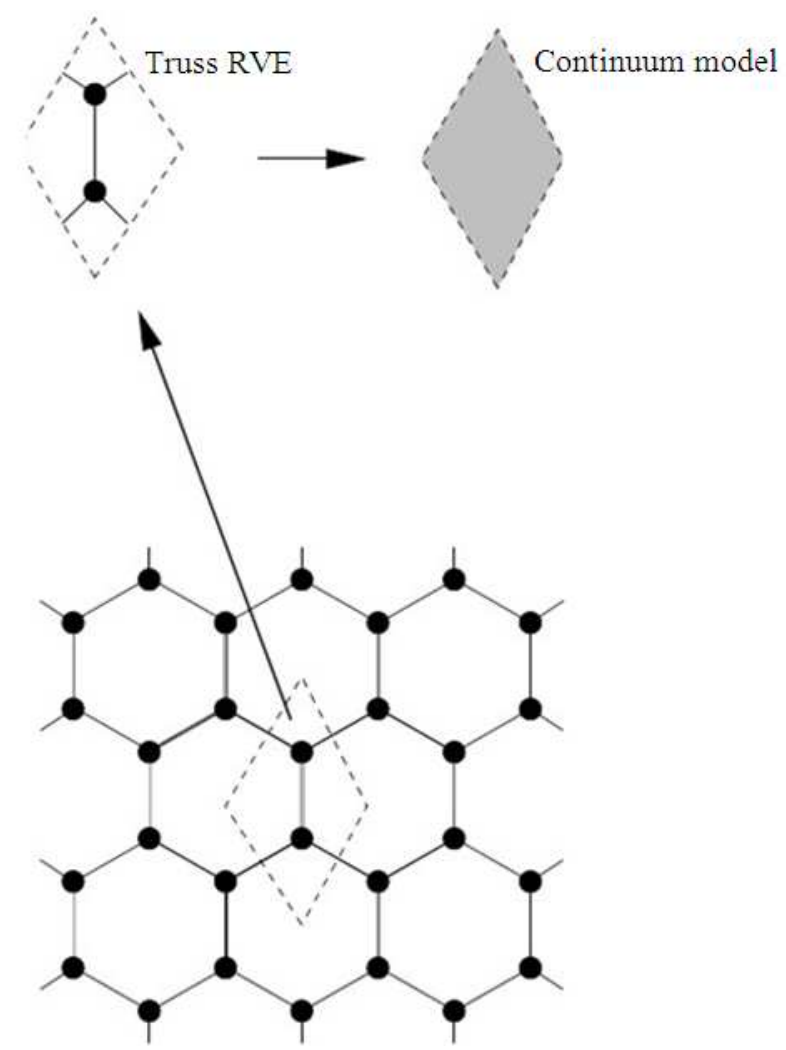

Fig. 5. Truss and continuum Representative Volume Element (RVE)

So we apply the shear load $\mathrm{N}_{12}$ (Fig. 6c), by the finite element method: (SAP, 2000) program, the nodal displacements are given and finally the kinematic variables $\mathrm{u}_{1,2}, \mathrm{u}_{2,1}$ and $\beta_{3}$ are obtained by the discrete element method then we apply the shear load $\mathrm{N}_{21}$ (Fig. 6b) and we obtain again $\mathrm{u}_{1,2}, \mathrm{u}_{2,1}$ and $\beta_{3}$. Finally, the resolution of the system of 2 equations for two parameters gives $\alpha$ and $\delta$. We follow the same way to search for the other parameters. The following values are found. Equation 30:

$$
\left\{\begin{array}{l}
\alpha=190.12\left[\frac{N}{m}\right] \\
\eta=1046.59\left[\frac{N}{m}\right] \\
L=345.29\left[\frac{N}{m}\right] \\
\varphi=3.482 \times 10^{6}[\mathrm{Nm}] \\
\phi=3.482 \times 10^{6}[\mathrm{Nm}] \\
v=8085[N m] \\
\Delta=\left[\begin{array}{cc}
0 & 0.89 \times 10^{11} \\
-0.89 \times 10^{11} & 0
\end{array}\right]\left[\frac{N}{m}\right]
\end{array}\right.
$$

\subsection{Discussion}

From the values of the material constants, we remark that the matrix $\mathrm{N}$ is neither symmetric nor skew-symmetric and the contribution of the rotation to the normal load might be considerable. Compared to the in-plane moment, for the Carbon nanotube case the drilling rotation induces a drilling moment which is not negligible.

\section{EXAMPLES}

The examples of the torsion of a straight SWNT tube and a straight composite tube made of polyimide reinforced with SWNT are studied. In both cases, SWNT layer is modeled with the proposed cosserat-type theory. The results illustrate the applicability of the proposed plate theory and the effect of the drilling rotation on the torsional rigidities and displacements. Comparisons with the torsional rigidities and displacements obtained with classical Cauchy-de Saint-Venant media, are presented.

\subsection{Torsion of SWNT}

A straight SWNT beam of axis (x) and length 1 is subjected to a uniform load $N_{\theta x}^{A} \vec{e}_{\theta}$ at its end $(\mathrm{x}=1)$ (Fig. 7), while the $y$ and $z$ displacements of the other end section $(x=0)$ are prevented. The beam has a uniform section which is delimited by closed circles.

The method of resolution starts similarly to the classical Cauchy-de Saint-Venant approach (Doghri, 2000). The displacement $\vec{U}$ of a material point is assumed to be the sum of an infinitesimal rotation $\theta^{\prime}$ in the $(\mathrm{y}, \mathrm{z})$ plane and an axial displacement $\mathrm{u}$ :

$$
\vec{U}(\bar{r}, \theta, x)=\bar{r} \theta^{\prime} \vec{e}_{\theta}+u \vec{e}_{x}
$$




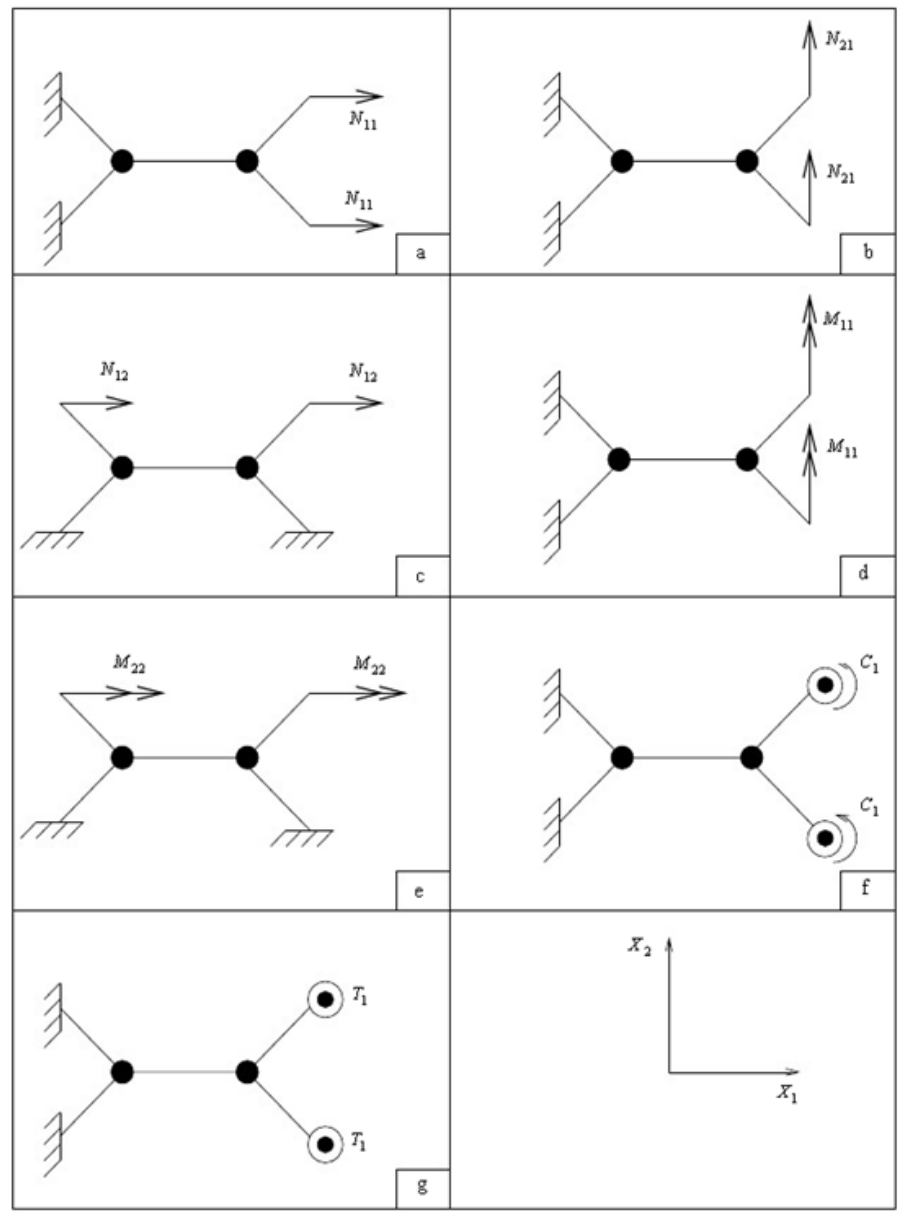

Fig. 6. Models for the determination of the elastic constants

where, $\bar{r}$ is the average radius equal to $\frac{R_{v}+R_{c}}{2}$

The infinitesimal rotation is assumed to be proportional to the axial distance $(\mathrm{x})$ of a cross section from the $(\mathrm{x}=0)$ section; i.e., $\theta^{\prime}=k x$, where $\mathrm{k}[1 / \mathrm{m}]$ is an angle of torsion per unit length.

It is supposed that the axial displacement is independent of (x), i.e., $u=k F(\bar{r}, \theta)$, where $F(\bar{r}, \theta)$ is a warping function to be determined.

According to these assumptions, the displacement is written as:

$\vec{U}(\bar{r}, \theta, x)=k \bar{r} x \vec{e}_{\theta}+k F(\bar{r}, \theta) \vec{e}_{x}$

We assume that the plate theory equations are valid for the thin tube by simple transformation into cylindrical coordinates, the mid-surface being described by $(\theta, x)$ coordinates. The strains are obtained by simple differentiation of the displacements. Equation 33:

$\varepsilon=\left[\begin{array}{cc}0 & \frac{1}{2}\left(\frac{1}{\bar{r}} \frac{\partial(k F)}{\partial \theta}+k \bar{r}\right) \\ \frac{1}{2}\left(\frac{1}{\bar{r}} \frac{\partial(k F)}{\partial \theta}+k \bar{r}\right) & 0\end{array}\right]$

The constitutive equations of section 3 give Equation 34:

$$
\begin{aligned}
& N=\alpha \varepsilon+\eta(t r \varepsilon) I+\beta_{r} \Delta \\
& =\left[\begin{array}{cc}
0 & \frac{\alpha}{2}\left(\frac{1}{\bar{r}} \frac{\partial(k F)}{\partial \theta}+k \bar{r}\right)+\beta_{r} \delta \\
\frac{\alpha}{2}\left(\frac{1}{\bar{r}} \frac{\partial(k F)}{\partial \theta}+k \bar{r}\right)-\beta_{r} \delta & 0
\end{array}\right]
\end{aligned}
$$



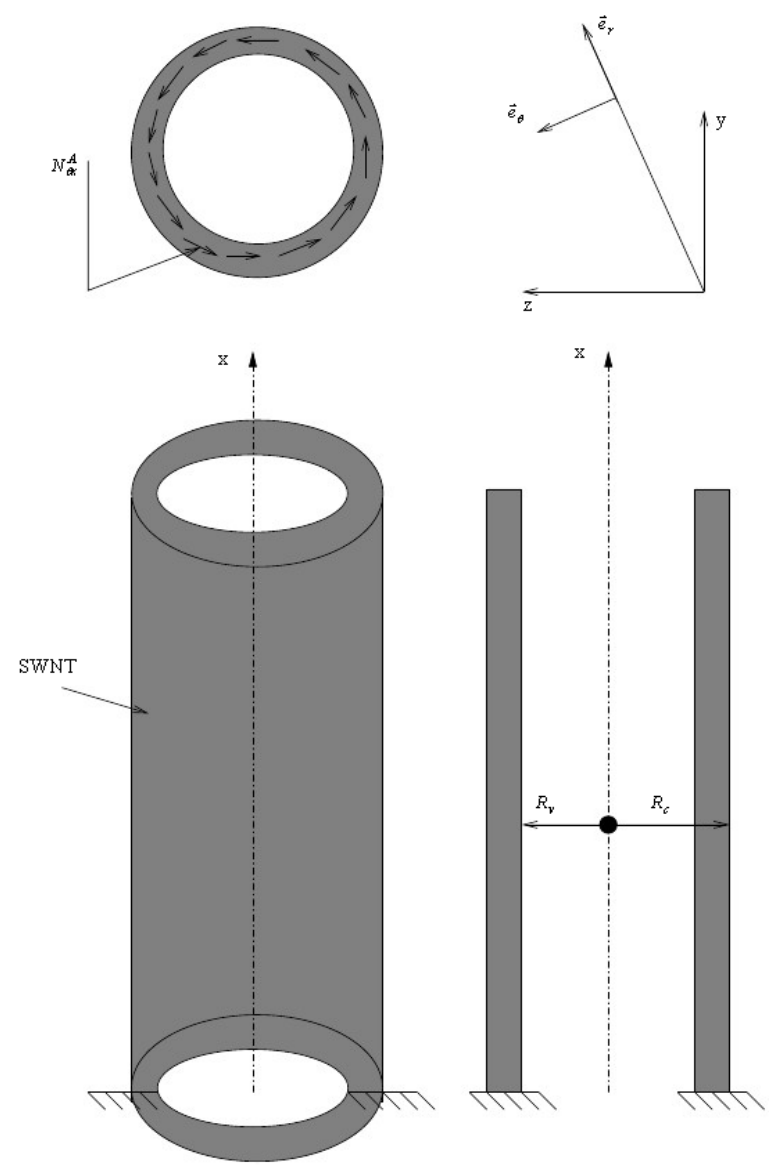

Fig. 7. Straight SWNT beam under $N_{\theta x}^{A}$ load

In the classical Kirchhoff-Love plate theory, the internal load tensor is written as:

$$
N=h\left[2 \mu \varepsilon+\frac{E v}{1-v^{2}} \operatorname{tr} \varepsilon\right]
$$

where, $\mathrm{h}$ designates the thickness of the tube.

Similarly, the equation for $\mathrm{N}$ of Equation 34 can be written as:

$$
N=h\left[2 \mu^{c} \varepsilon+\xi \operatorname{tr\varepsilon } I+\beta_{r} \Lambda\right]
$$

Where:

$$
\mu^{c}=\frac{\alpha}{2 h}, \xi=\frac{\eta}{h}, \Lambda=\frac{\Delta}{h}
$$

The equilibrium equation $\left(\operatorname{div} N+\vec{f}_{w}=0\right)$ gives Equation 36:

$$
\left\{\begin{array}{l}
\beta_{r}=\beta_{r}(\theta) \\
\frac{\alpha k}{2 \bar{r}} \frac{\partial^{2} F}{\partial \theta^{2}}-\delta \frac{\partial \beta_{r}}{\partial \theta}=0
\end{array}\right.
$$

On the end section $(\mathrm{x}=1)$, we shall write that the load applied is equal to $N_{\theta x}^{A}$ :

$$
N \vec{e}_{x}=N_{\theta x}^{A} \vec{e}_{\theta} \Rightarrow N_{\theta x}^{A}=\frac{\alpha}{2}\left(\frac{1}{\bar{r}} \frac{\partial(k F)}{\partial \theta}+k \bar{r}\right)+\beta_{r} \delta
$$

Thus the normal fort tensor can be written as:

$$
N=\left[\begin{array}{cc}
0 & N_{\theta x}^{A} \\
N_{x \theta}^{A}-2 \beta_{r} \delta & 0
\end{array}\right]
$$

In summary, the problem is the following: find a function $F(\bar{r}, \theta)$ which satisfies:

$$
\left\{\begin{array}{l}
\beta_{r}=\beta_{r}(\theta) \\
\frac{\alpha k}{2 \bar{r}} \frac{\partial^{2} F}{\partial \theta^{2}}-\delta \frac{\partial \beta_{r}}{\partial \theta}=0 \\
N_{\theta x}^{A}=\frac{\alpha}{2}\left(\frac{k}{\bar{r}} \frac{\partial F}{\partial \theta}+k \bar{r}\right)+\beta_{r} \delta
\end{array}\right.
$$

The system (39) shows that the drilling rotation $\beta_{\mathrm{r}}$ is constant.

The Equation 39 gives Equation 40:

$F=\left[\frac{2 \bar{r}}{\alpha k}\left(N_{\theta x}^{A}-\beta_{r} \delta\right)-\bar{r}^{2}\right] \theta+F_{1}(\bar{r})$

For a circular cylindrical tube, there is no warping in the classical Cauchy-de Saint-Venant solution. Similarly in the case of Cosserat medium, we assume that the warping function is nil:

$F=0 \Rightarrow \beta_{r}=\frac{N_{\theta x}^{A}-\frac{\alpha k \bar{r}}{2}}{\delta}$ and $\quad F_{1}(\bar{r})=0$

The stress vector resultant over the Carbon phase thickness is:

$$
N_{\theta x}^{A}=\int_{R_{v}}^{R_{c}} \sigma_{\theta x} d r
$$


For a circular cylindrical tube, in the classical Cauchy-de Saint-Venant solution, the stress $\sigma_{\theta \mathrm{x}}$ is equal to $k \mu r$. In the Cosserat medium, which differs from the Cauchy one in the drilling rotation, in addition to the stress due to the symmetric strain $\varepsilon$, there is stress related to the drilling rotation. Therefore, we assume that the stress $\sigma_{\theta \mathrm{x}}$ can be split in two parts:

$$
\sigma_{\theta x}=\sigma_{\theta x}^{l}+\sigma_{\theta x}^{2}
$$

Where:

$$
\begin{array}{ll}
\sigma_{\theta x}^{l}=k \mu^{c} r & =\text { The stress due to the symmetric strain } \varepsilon \\
\sigma_{\theta x}^{2} & =\text { The stress related to the drilling rotation }
\end{array}
$$

Because $N_{\theta x}^{A}=$ Constant, $\sigma_{\theta x}^{2}$ only depends on the coordinate (r), hence it can be taken equal to $k c f(r)$ Therefore:

$$
\sigma_{\theta x}=\sigma_{\theta x}^{l}+\sigma_{\theta x}^{2}=k \mu^{c} r+k c f(r)
$$

where, the constant $c$ and the function $f(r)$ are to be determined.

The applied tangential load is:

$$
\begin{aligned}
& N_{\theta x}^{A}=\int_{R_{v}}^{R_{c}}\left(k \mu^{c} r+k c f(r)\right) d r \\
& \left.=\frac{k \mu^{c}}{2}\left(R_{c}^{2}-R_{v}^{2}\right)+k c \int_{R_{v}}^{R_{c}} f(r)\right) d r
\end{aligned}
$$

From Equation 41:

$$
N_{\theta x}^{A}=k \alpha \frac{\bar{r}}{2}+\beta_{r} \delta=k \mu^{c} h \bar{r}+\beta_{r} \delta=\frac{k \mu^{c}}{2}\left(R_{c}^{2}-R_{v}^{2}\right)+\beta_{r} \delta
$$

Which implies that Equation 45:

$$
\beta_{r}=\frac{k c}{\delta} \int_{R_{v}}^{R_{c}} f(r) d r
$$

Knowing that the torque per unit length of the average circle due to the tangential load $\mathrm{N}_{\theta \mathrm{x}}$ is $N_{\theta x} \cdot 2 \bar{r}$ thus the resultant torque is $N_{\theta x} \cdot 2 \bar{r} \cdot 2 \pi \bar{r}$.

The torsional rigidiy is then:

$$
\begin{aligned}
\frac{M}{k} & =\frac{N_{\theta x}^{A} \cdot 2 \bar{r} \cdot 2 \pi \bar{r}}{k} \\
& =\pi\left(R_{v}+R_{c}\right)^{2}\left[\frac{\mu^{c}}{2}\left(R_{c}^{2}-R_{v}^{2}\right)+c \int_{R_{v}}^{R_{c}} f(r) d r\right]
\end{aligned}
$$

The stress $\sigma_{\theta x}^{2}$ related to the drilling rotation is assumed to be parabolic (Fig. 8). Equation 47:

$$
\sigma_{\theta x}^{2}=k c f(r)=k c\left(r^{2}-\left(R_{v}+R_{c}\right) r-R_{v} R_{c}\right)
$$

The constant $\mathrm{c}$ is computed by equating the values of $\frac{\sigma_{\theta x}}{k}$ for Cauchy and Cosserat solution for a given value of $\bar{r}=\bar{r}_{0}$ chosen rather large but physically acceptable. This gives Equation 48:

$c=\frac{4}{h^{2}}\left(\mu^{c}-\mu\right) \bar{r}_{0}$

Therefore the torsional rigidity and the drilling rotation are obtained as follows. Equation 49 and 50:

$\frac{M}{k}=\pi\left(R_{v}+R_{c}\right)^{2}$
$\left[\frac{\mu^{c}}{2}\left(R_{c}^{2}-R_{v}^{2}\right)+c\left(\frac{R_{v}^{3}-R_{c}^{3}}{6}+\frac{R_{v} R_{c}^{2}-R_{c} R_{v}^{2}}{2}\right)\right]$

And:

$\beta_{r}=\frac{k c}{\delta}\left[\left(\frac{R_{v}^{3}-R_{c}^{3}}{6}+\frac{R_{v} R_{c}^{2}-R_{c} R_{v}^{2}}{2}\right)\right]$

When the drilling rotation is nil, the stress due to $\beta_{\mathrm{r}}$ is also nil, one has Equation 51:

$\frac{M}{k}=\pi \frac{\mu^{c}}{2}\left(R_{v}+R_{c}\right)^{2}\left(R_{c}^{2}-R_{v}^{2}\right)$

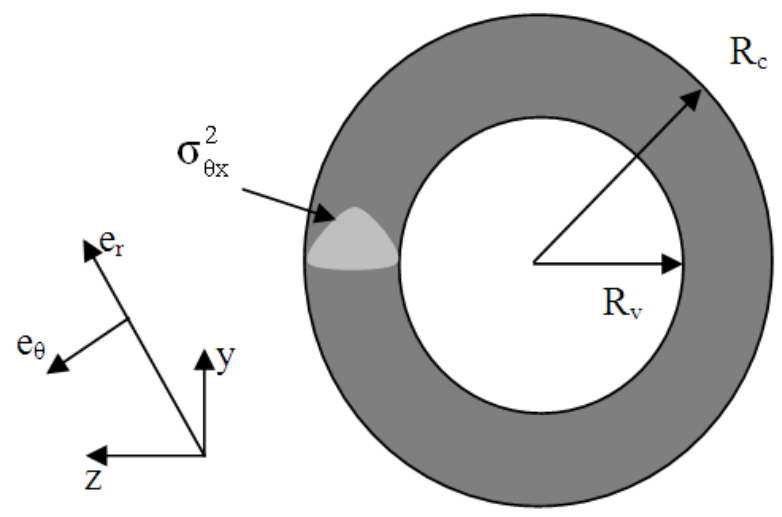

Fig. 8. Representation of the stress related to the drilling rotation 
The expression (51) corresponds to that of SWNT considered as a Cauchy medium.

\subsection{Torsion of SWNT/Polyimide Composite Tube}

We now consider the example of a straight composite tube made of polyimide reinforced with SWNT. The graphene layer is modeled as a Cosserat medium (Fig. 9). The composite tube of axis (x) and length 1 is subjected to a uniform load $N_{\theta x}^{A} \vec{e}_{\theta}$ at its end $(\mathrm{x}=1)$, while the $\mathrm{y}$ and $\mathrm{z}$ displacements of the other end section $(x=0)$ are prevented. The tube has a uniform section which is delimited by closed circle.

Adopting the cylindrical coordinates, the stress tensors in the matrix and the Carbon phases are taken respectively:

$$
\begin{aligned}
\sigma^{m} & =\left[\begin{array}{cc}
0 & k^{m} \mu^{m} r \\
k^{m} \mu^{m} r & 0
\end{array}\right] \\
\sigma^{c} & =\left[\begin{array}{cc}
0 & \sigma_{\theta x} \\
\sigma_{x \theta} & 0
\end{array}\right]
\end{aligned}
$$

Using the results of section 4.1, the stresses in the Carbon layer are:

$\sigma_{\theta x}=k^{c} \mu^{c} r+k^{c} c\left[r^{2}-\left(R_{v}+R_{c}\right) r+R_{v} R_{c}\right]$

In the matrix phase, the strains are given by isotropic linear elasticity as:

$$
\varepsilon^{m}=\left[\begin{array}{cc}
0 & k^{m} \frac{r}{2} \\
k^{m} \frac{r}{2} & 0
\end{array}\right]
$$

The matrix displacement is:

$$
\vec{u}^{m}=k^{m} r x \vec{e}_{\theta}
$$

The continuity of the traction at the matrix/Carbon interface is satisfied:

$$
\left[\sigma^{c}-\sigma^{m}\right] \vec{e}_{r}=\overrightarrow{0}
$$

The Carbon phase displacement is taken as:

$$
\vec{u}^{c}=k^{c} r x \vec{e}_{\theta}
$$

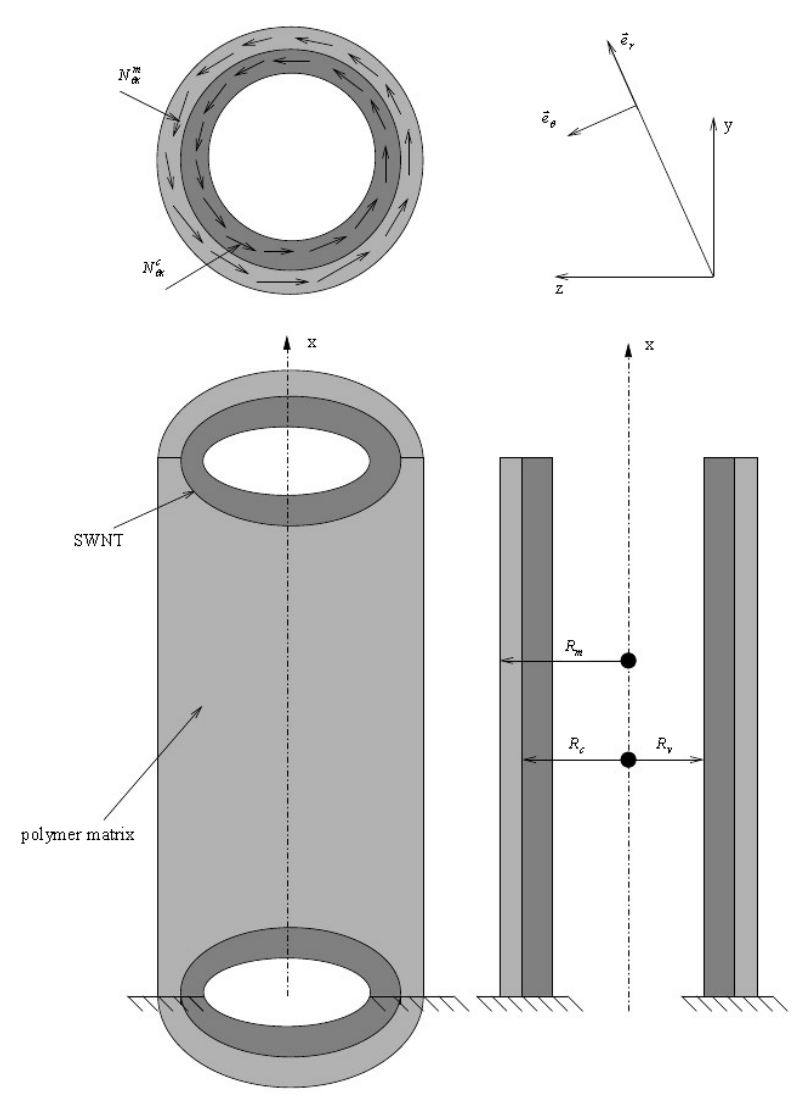

Fig. 9. Straight SWNT composite tube under $N_{\theta x}^{A}$ load

At $\mathrm{r}=\mathrm{R}_{\mathrm{c}}$, the displacement continuity at the matrix/Carbon interface implies Equation 59:

$k^{m}=k^{c}=k$

So, in the Carbon phase, the strains are derived as:

$\varepsilon=\left[\begin{array}{cc}0 & k \frac{r}{2} \\ k \frac{r}{2} & 0\end{array}\right]$

The stress vector (traction) on any cross section of outward normal $\vec{e}_{x}$ is $\vec{t}^{i}=\sigma_{\theta x}^{i} \vec{e}_{\theta}$, with $\mathrm{i}=\mathrm{c}$ or $\mathrm{m}$. It is easily checked that its resultant is nil. The resultant of the stress vector over the thickness of the Carbon layer gives: 
$\int_{R_{v}}^{R_{c}} \vec{t}^{c} d r=\left[\frac{k \mu^{c}}{2}\left(R_{c}^{2}-R_{v}^{2}\right)+k c\left(\frac{R_{v}^{3}-R_{c}^{3}}{6}+\frac{R_{v} R_{c}^{2}-R_{c} R_{v}^{2}}{2}\right)\right] \vec{e}_{\theta}$

$=N_{\theta x}^{A c} \vec{e}_{\theta}$

where, $N_{\theta x}^{A c}[N / m]$ is the load applied to the SWNT phase.

The resultant of the stress vector over the matrix thickness is:

$$
\begin{aligned}
& \int_{R_{c}}^{R_{m}} \overrightarrow{\vec{t}}^{m} d r=\frac{k \mu^{m}}{2}\left(R_{m}^{2}-R_{c}^{2}\right) \vec{e}_{\theta} \\
& =N_{\theta x}^{A m} \vec{e}_{\theta}
\end{aligned}
$$

where, $N_{\theta x}^{A m}[N / m]$ is the load applied to the matrix phase.

The torque applied to the Carbon phase is:

$$
M^{c}=\pi\left(R_{c}+R_{v}\right) N_{\theta x}^{A c}
$$

And the torque applied to the matrix phase is:

$$
M^{m}=\pi\left(R_{c}+R_{m}\right) N_{\theta x}^{A m}
$$

Then, the composite beam rigidity is:

$$
\begin{aligned}
& \frac{M}{k}=\frac{M^{c}+M^{m}}{k}= \\
& \pi\left(R_{v}+R_{c}\right)^{2}\left[\frac{\mu^{c}}{2}\left(R_{c}^{2}-R_{v}^{2}\right)+c\left(\frac{R_{v}^{3}-R_{c}^{3}}{6}+\frac{R_{v} R_{c}^{2}-R_{c} R_{v}^{2}}{2}\right)\right] \\
& +\pi \frac{\mu^{m}}{2}\left(R_{c}+R_{m}\right)^{2}\left(R_{m}^{2}-R_{c}^{2}\right)
\end{aligned}
$$

Note that $\mu^{\mathrm{m}}$ is the classical elastic shear modulus for the matrix phase but for the Carbon layer $\mu^{\mathrm{c}}$ is not the classical elastic shear modulus and is defined in Equation 35.

In the case of a nil drilling rotation, the associated stress is also nil so that $\mathrm{c}=0$ and:

$$
\begin{aligned}
& \frac{M}{k}=\frac{\pi}{2} \\
& {\left[\left(R_{v}+R_{c}\right)^{2} \mu^{c}\left(R_{c}^{2}-R_{v}^{2}\right)+\mu^{m}\left(R_{c}+R_{m}\right)^{2}\left(R_{m}^{2}-R_{c}^{2}\right)\right]}
\end{aligned}
$$

It is the expression obtained when the composite phases are considered as Cauchy media.

\section{NUMERICAL SIMLULATIONS}

A polyimide (LaRC-SI) is reinforced with fully aligned, long homogeneously dispersed SWNT; see Table 1 for the properties when the graphene layer is considered as Cauchy medium. In the case of Cosserat medium, the mechanical properties are given in section 3. For both approaches, the predicted mechanical response of the composite is reported in Fig. 10 to 18. In all figures the SWNT thickness is kept constant equal to $0.34 \mathrm{~nm}$. If the $\mathrm{x}$-axis is the SWNT volume fraction, the internal radius $R_{v}$ is kept constant equal to $2 \mathrm{~nm}$ and it is the polyimide tube radius $R_{m}$ which varies. But if the $x$-axis is the internal radius $R_{v}$, the SWNT volume fraction is taken equal to $3 \%$.

\section{Interpretation}

From Fig. 10 we conclude that the Cosserat normalized torsional rigidity, which is equal to the Cosserat torsional rigidity normalized by the Cauchy torsional one $(\mathrm{M} / \mathrm{k})^{\mathrm{c}} /(\mathrm{M} / \mathrm{k})^{\mathrm{c}}$, changes with the internal radius so the figure shows a size effect on the torsional rigidity.

Figure 11 plots the variation of the Cauchy and the Cosserat composite torsional rigidities with the internal radius. It shows that for large values of internal radius where the plate theory is applicable, the Cauchy torsional rigidity is larger than that of the Cosserat one. This torsional rigidity decrease is due to the addition of the drilling rotation DOF.

Figure 12 and 13 plot respectively the variation of the torsional rigidities and the normalized torsional rigidity with the SWNT volume fraction. The two figures show clearly that for low volume fraction, the Cosserat solution is close to the Cauchy one.

Figure 14 plots the variation of $\sigma_{\theta x} / k$ in a point of the Carbon phase mid-surface with internal radius, it indicates that $\sigma_{\theta \mathrm{x}} / \mathrm{k}$ in the graphene layer considered as Cosserat medium is higher than that in the graphene layer taken as Cauchy medium.

Figure 15 and 16 show respectively the variation of the tangential displacement of a material point in the Carbon phase mid-surface normalized by the average diameter of the SWNT tube $u_{\theta} / d, d=R_{v}+R_{c}$, as a function of SWNT volume fraction and as a function of the axial distance.

Tangential displacements in these figures are obtained by applying a $10^{-10} \mathrm{nNm}$ torque, for the Fig. 15, the displacement is calculated in $0.5 \mathrm{~mm}$ axial distance. 
Abdellatif Selmi et al. / American Journal of Applied Sciences 11 (8): 1255-1273, 2014

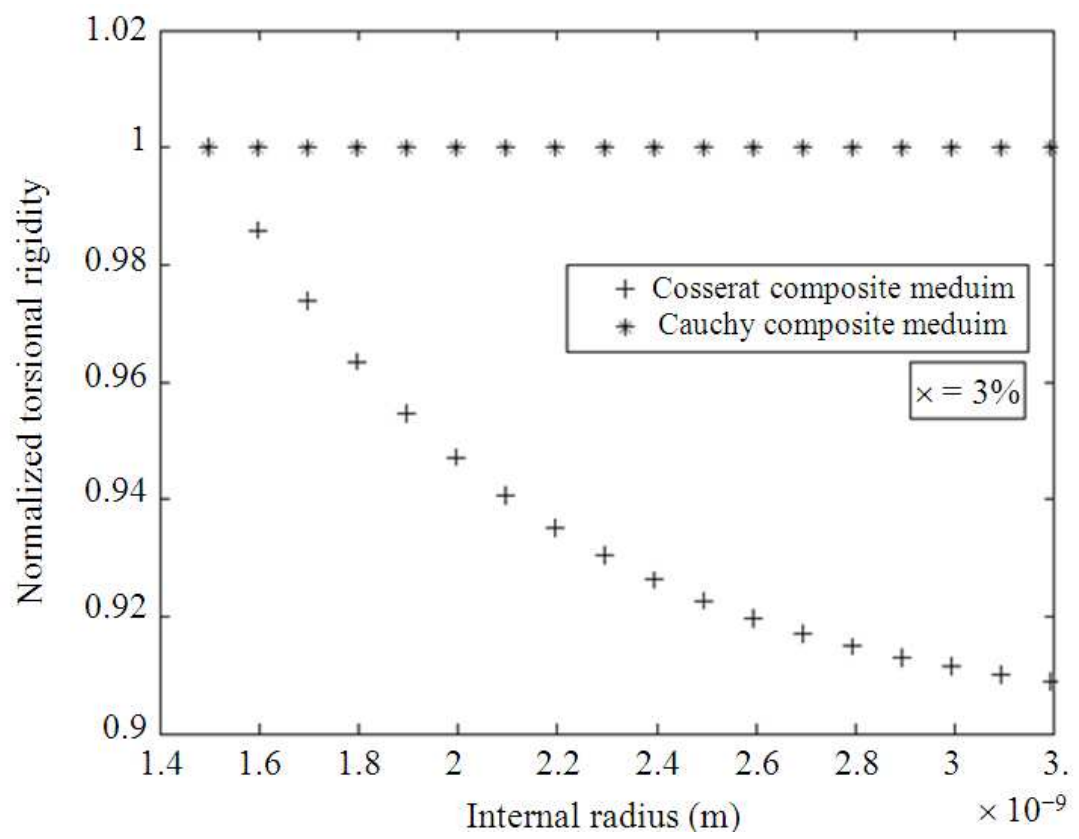

Fig. 10. SWNT/LaRC-SI composite. Variation of the normalized torsional rigidity with the internal radius: Comparison between results of Cauchy-de Saint-Venant and proposed Cosserat theories

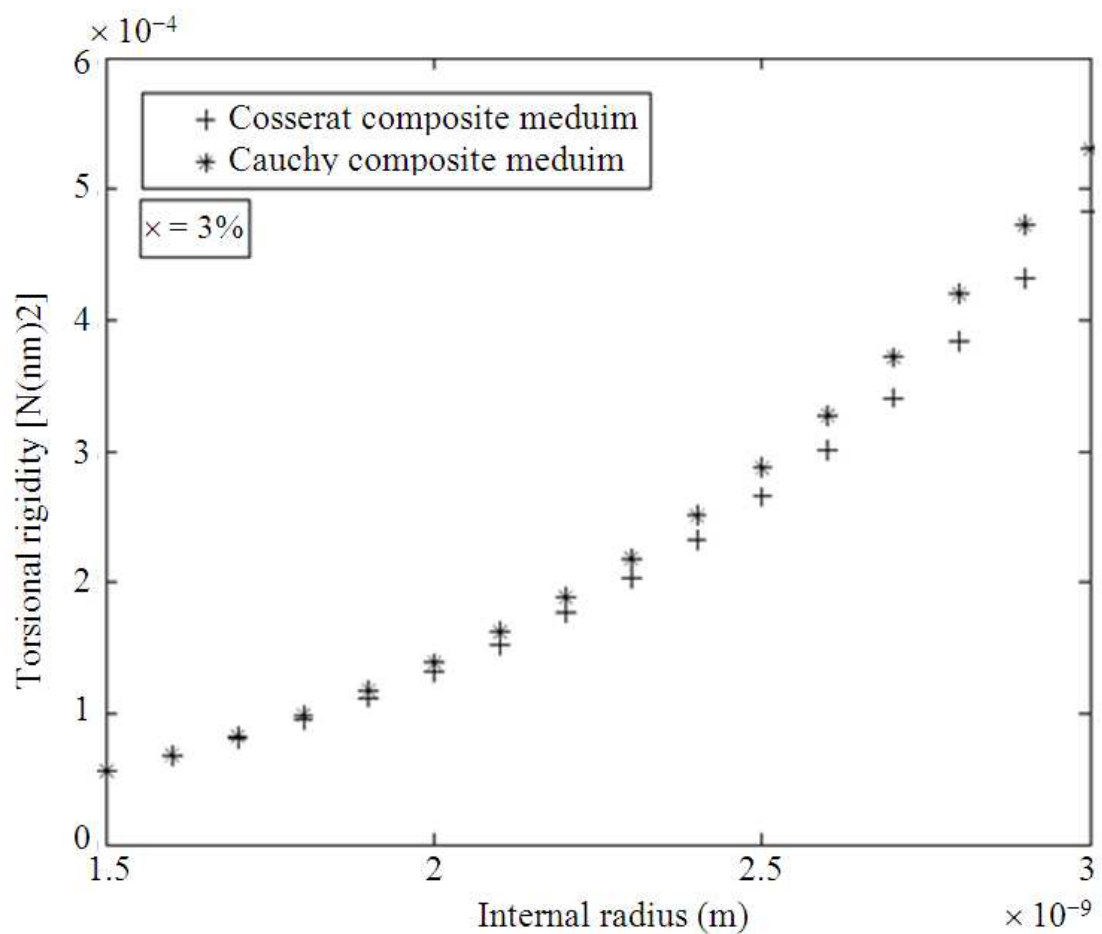

Fig. 11. SWNT/LaRC-SI composite. Variation of the torsional rigidity with the internal radius: Comparison between the Cauchy-de Saint-Venant and Cosserat theories 
Abdellatif Selmi et al. / American Journal of Applied Sciences 11 (8): 1255-1273, 2014

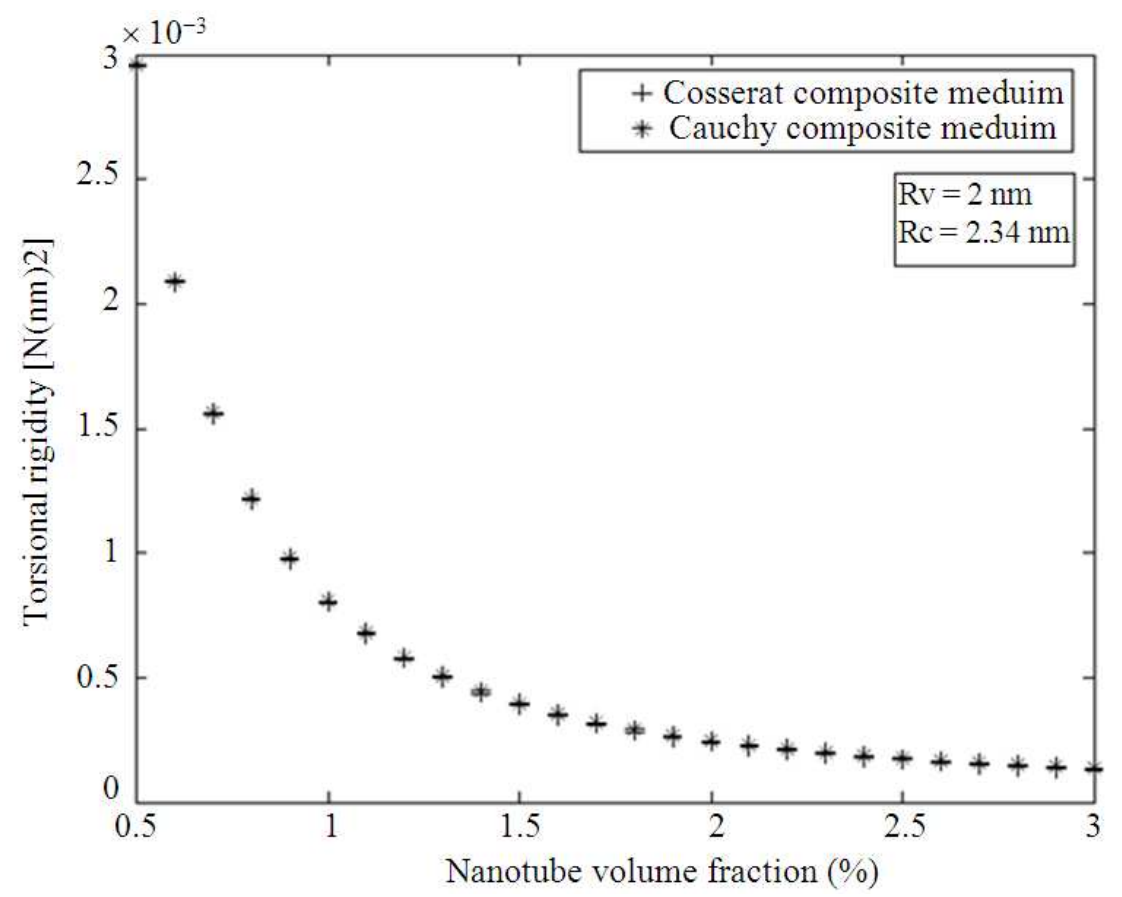

Fig. 12. SWNT/LaRC-SI composite. Variation of the torsional rigidity with the SWNT volume fraction: Comparison between the cauchy-de saint-venant and cosserat theories

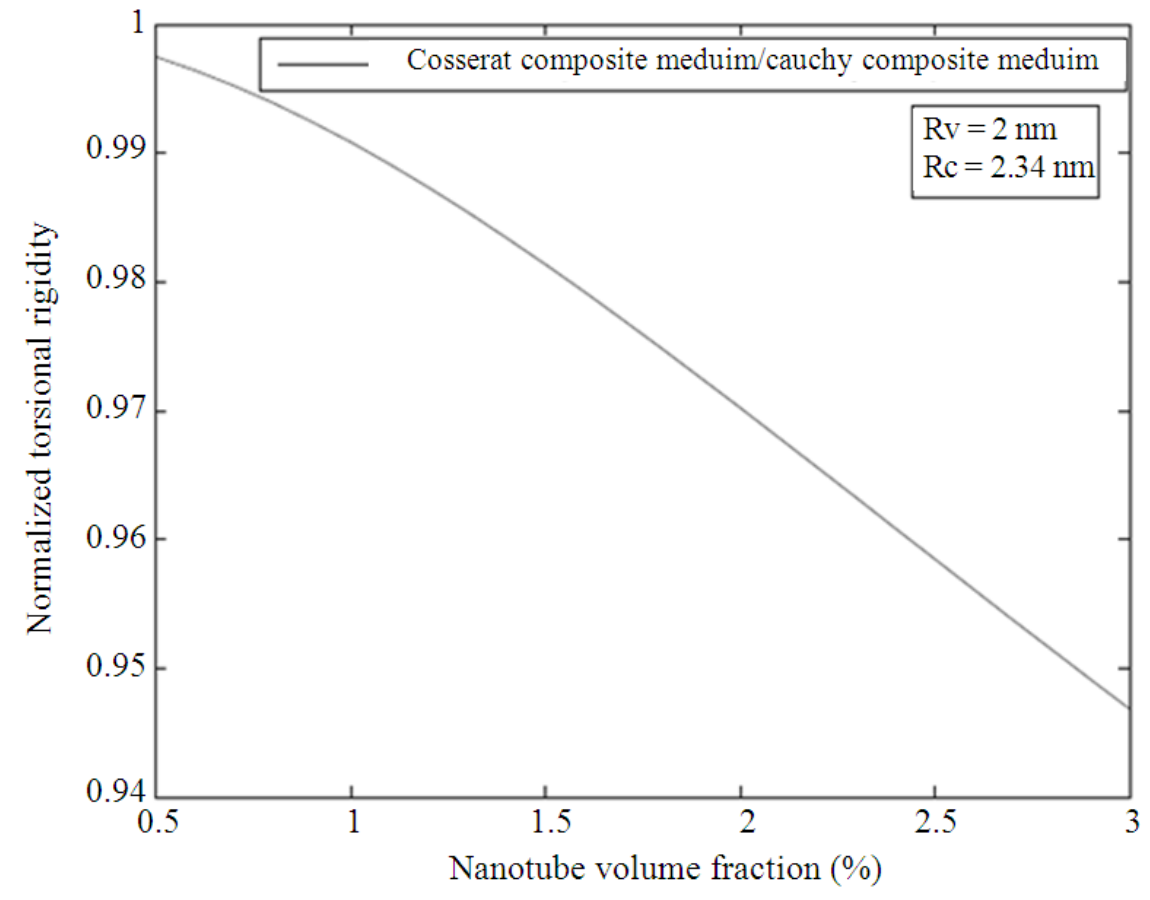

Fig. 13. SWNT/LaRC-SI composite. Variation of the normalized torsional rigidity with the SWNT volume fraction 
Abdellatif Selmi et al. / American Journal of Applied Sciences 11 (8): 1255-1273, 2014

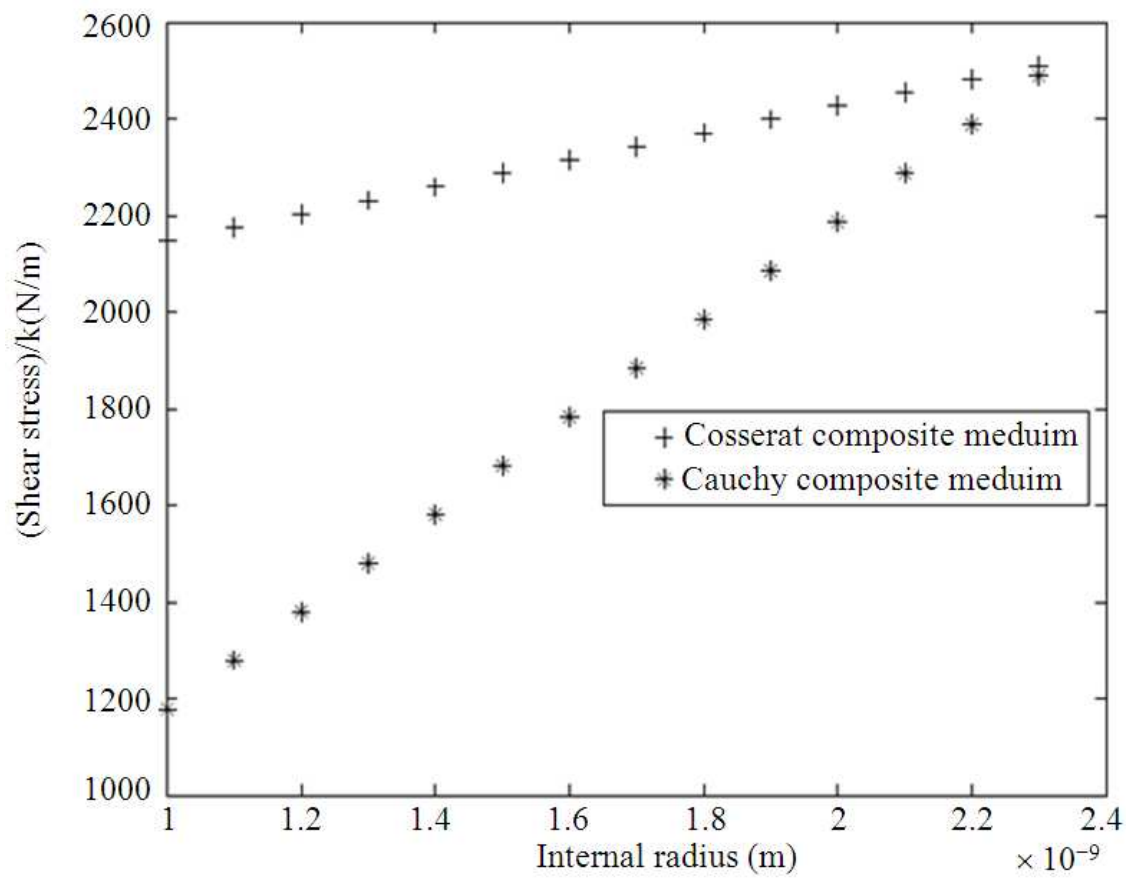

Fig. 14. SWNT/LaRC-SI composite. Shear stress/k: Comparison between the cauchy-de saint-venant and cosserat theories

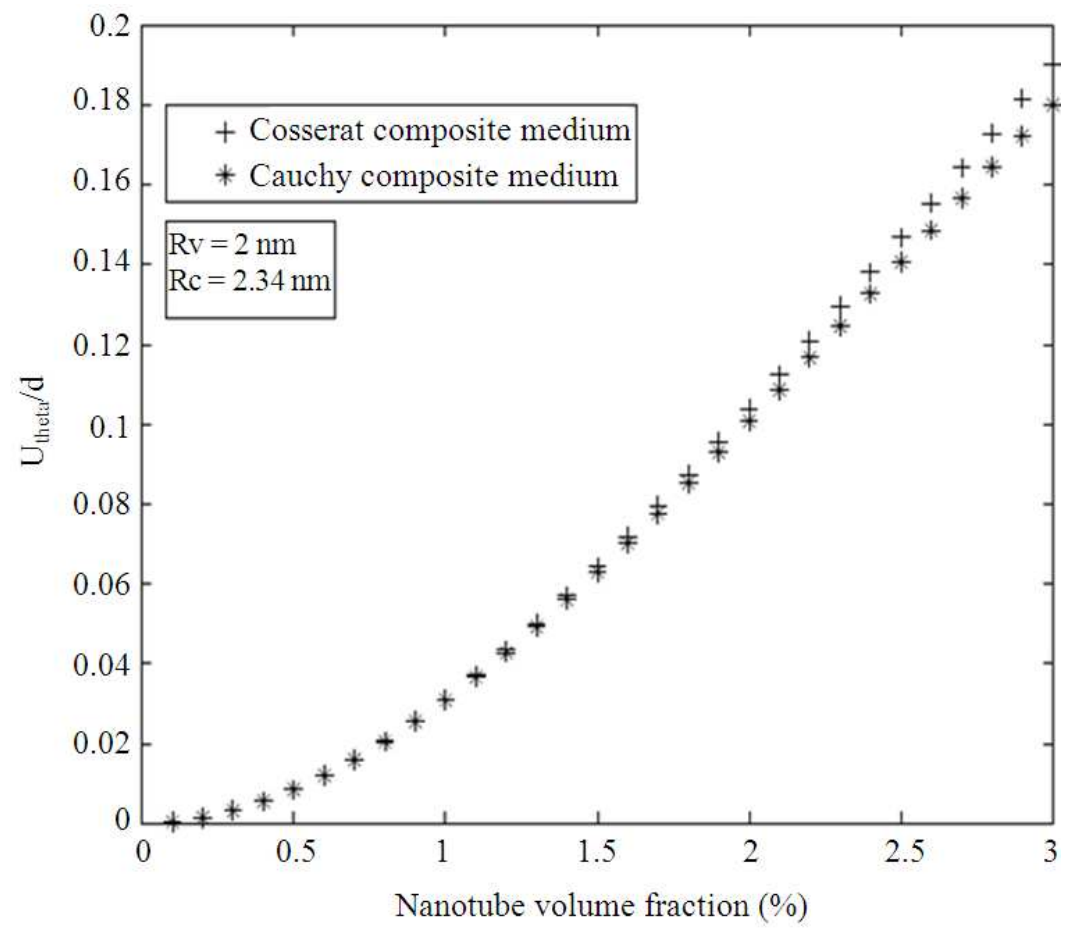

Fig. 15. SWNT/LaRC-SI composite. Variation of the normalized tangential displacement with SWNT volume fraction: Comparison between the cauchy-de saint-venant and cosserat theories 
Abdellatif Selmi et al. / American Journal of Applied Sciences 11 (8): 1255-1273, 2014

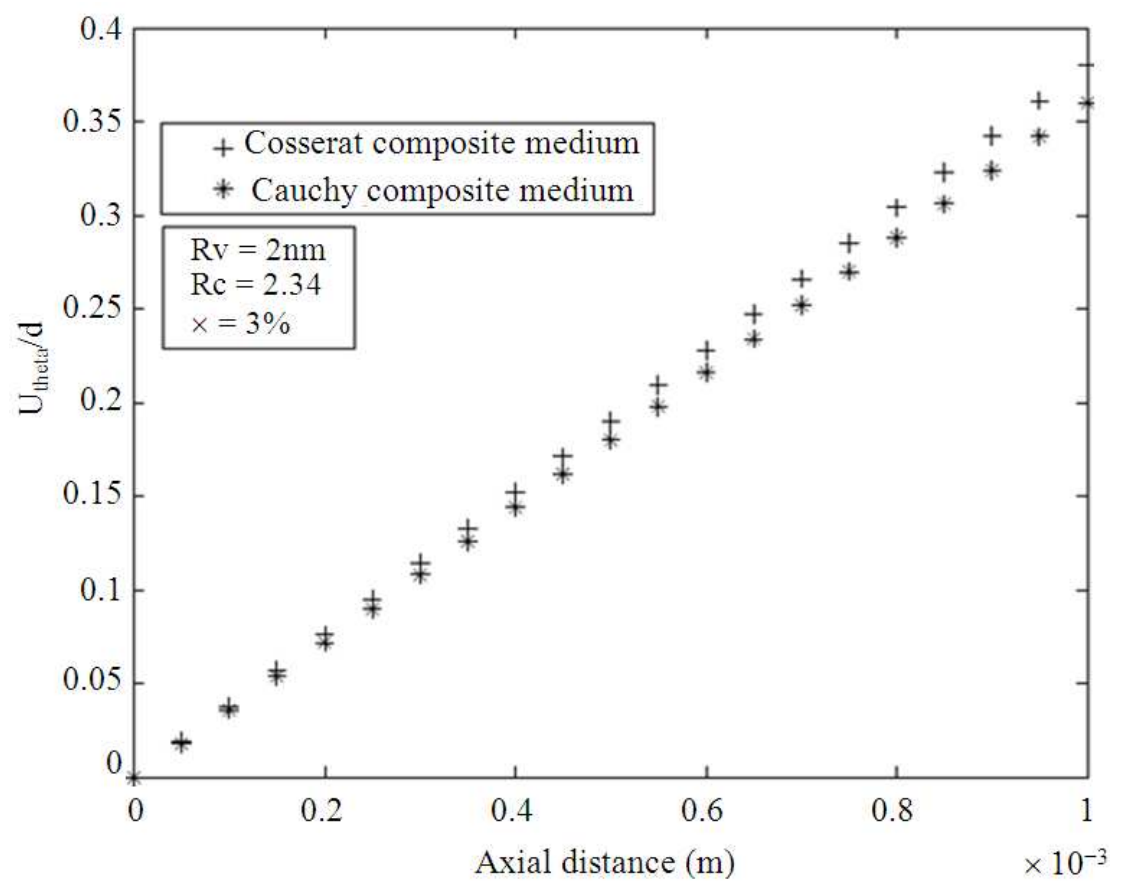

Fig. 16. SWNT/LaRC-SI composite. Variation of the normalized tangential displacement with the axial distance: Comparison between the cauchy-de saint-venant and cosserat theories

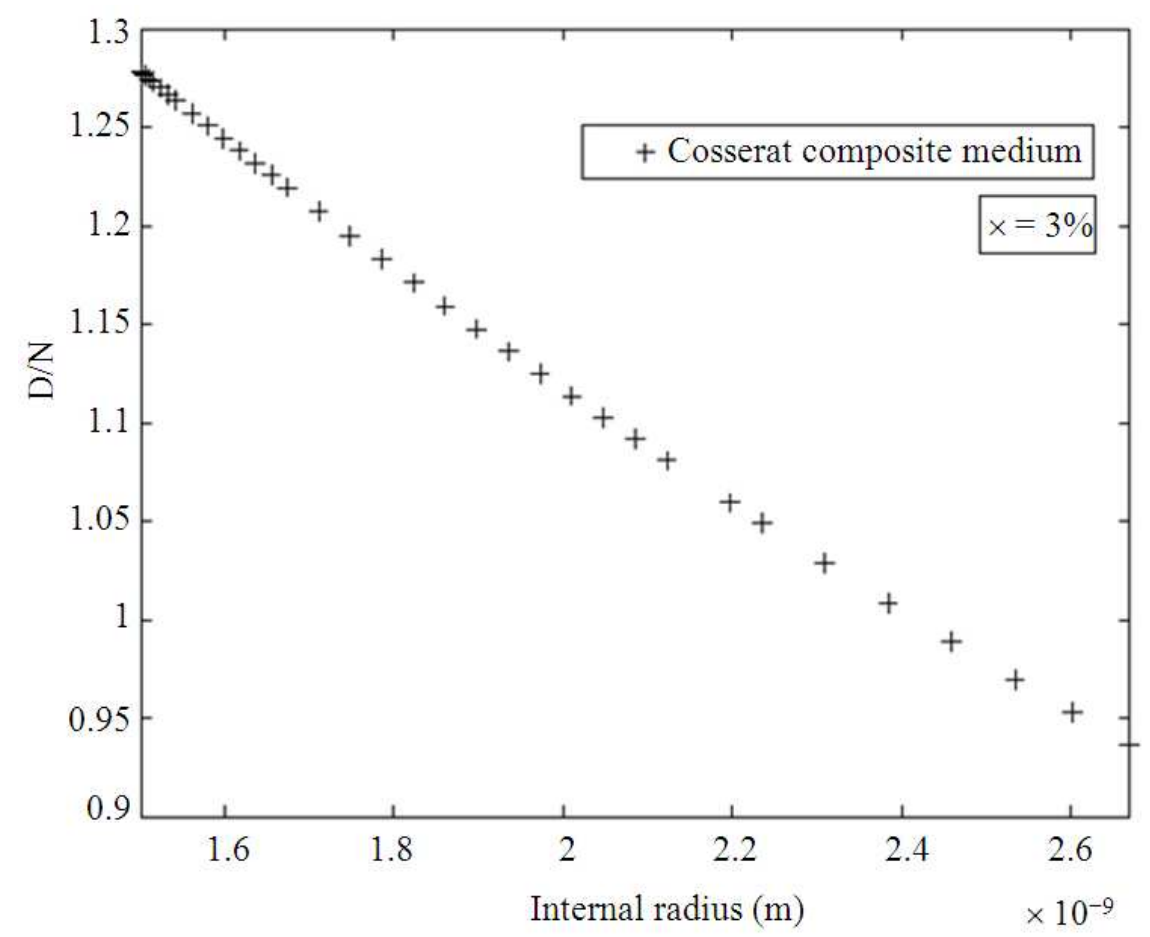

Fig. 17. SWNT/LaRC-SI composite. Variation of the normalized D parameter by the tangential load with the internal radius 


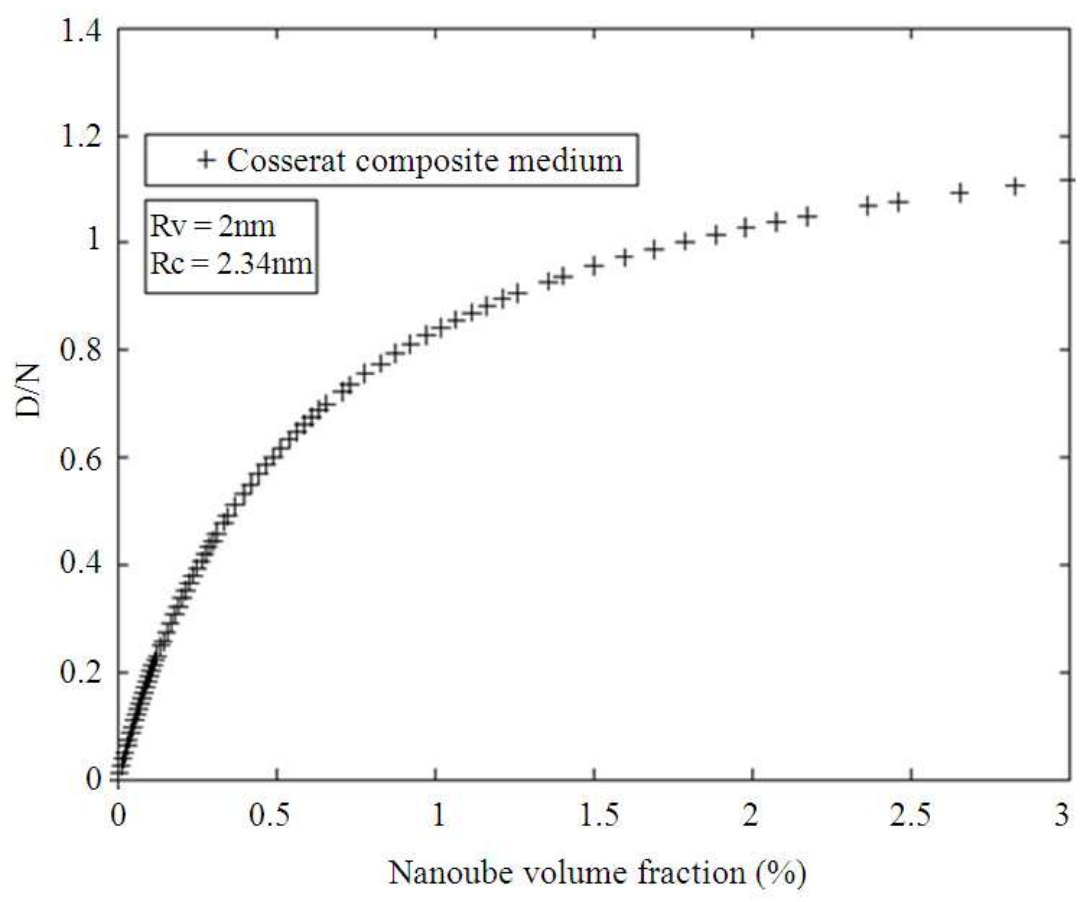

Fig. 18. SWNT/LaRC-SI composite. variation of the normalized D parameter by the tangential load with the SWNT vo-lume fraction

Table 1. Elastic constants of LaRC-SI and Continuum graphene, after Selmi et al. (2007)

\begin{tabular}{lcl}
\hline & $\begin{array}{c}\text { Young's } \\
\text { modulus }(\mathrm{GPa})\end{array}$ & $\begin{array}{l}\text { Poisson's } \\
\text { ratio }\end{array}$ \\
\hline LaRC-SI & 3.8 & 0.4000 \\
Continuum graphene & 2520.0 & 0.25 \\
\hline
\end{tabular}

The figures show that the normalized displacement associated to the Cosserat composite medium is a little higher than that associated to the Cauchy composite medium; this is predictable and it is due to the torsional rigidity difference.

Knowing that for a fixed internal radius $R_{v}$, the rotation $\beta_{\mathrm{r}}$ is constant and knowing that there is no drilling moment applied to the SWNT/Polyimide composite, Equation 19d is reduced to $\mathrm{D}=0$.

Figure 17 and 18 show respectively the variation of the parameter $\mathrm{D}$, equal to $2 \delta \beta_{\mathrm{r}}$, normalized by the tangential load with respect to the SWNT volume fraction and the internal radius.

These figures prove that for low SWNT volume fraction and for large values of internal SWNT radius, the parameter $\mathrm{D}$ is near to zero and explain the fact that not taking the equilibrium Equation 19d into account is not a bad estimation, hence all equilibrium equations are satisfied.

\section{LIMITATION AND FUTURE RESEARCH}

We bring to attention that Equation $19 \mathrm{~d}$ is not verified because taking it into account leads to a complicated problem which needs supplementary boundary conditions. But it was seen that when the tangential load value is not too large, this equation is basically satisfied.

The developed Cosserat plate theory can be used in future research to clarify the behaviour of granular and large molecular materials such as polymers in which the transmission of interaction may be not only through a force vector but also through a couple or moment vector. The developed theory coupled with micromechanical techniques can be adopted in order to investigate the size effects which cannot be detected by classical homogenization procedures.

\section{SUMMARY AND CONCLUSION}

In order to study the angle variation phenomenon effect on the SWNT mechanical behaviour, a Cosserattype elastic plate theory based on the virtual principle work was developed. For this theory which takes into account the drilling rotations, the internal loads, the 
equilibrium equations, the boundary conditions and the linear elastic constitutive equations were derived. The application of this approach to a nanotube microstructure and a SWNT/Polyimide composite under torsion proves its capability and shows a size effect on the torsional rigidity.

\section{REFERENCES}

Ambartsumian, S.A., 2002. Nontraditional theories of shells and plates. Applied Mechan. Rev., 55: 35-44. DOI: $10.1115 / 1.1495002$

Atluri, S.N., 1984. Alternate stress and conjugate strain measures and mixed variational formulations involving rigid rotations, for computational analysis of finitely deformed solids, with application to plates and shells-I theory. Composite Structures, 18: 93116. DOI: 10.1016/0045-7949(84)90085-3

Doghri, I., 2000. Mechanics of Deformable Solid-Linear, Nonlinear, Analytical and Computational Aspects. Springer-Verlag, Berlin. DOI: 10.1007/978-3-66204168-0

Erbay, H.A., 1997. On the asymptotic membrane theory of thin hyperelastic plates. Int. J. Eng. Sci., 35: 151170. DOI: 10.1016/S0020-7225(96)00068-7

Erbay, H.A., 2000. An asymptotic theory of thin micropolar plates. Int. J. Eng. Sci., 38: 1497-1516. DOI: 10.1016/S0020-7225(99)00118-4

Eringen, A.C., 1967. Theory of micropolar plates. J. Applied Math. Physics, 18: 12-30. DOI: 10.1007/BF01593891

Kreja, I. and R. Schmidt, 2006. Large rotations in firstorder shear deformation FE analysis of laminated shells. Int. J. Non-Linear Mechan., 41: 101-123. DOI: 10.1016/j.ijnonlinmec.2005.06.009

Kumar, S., T.D Dang, F.E. Arnold, A.R. Bhattacharyya and B.G. Min et al., 2002. Synthesis, structure and properties of $\mathrm{PBO} / \mathrm{SWNT}$ composites. Macromolecules, 35: 9039-9043. DOI: 10.1021/ma020505

Meoli, D. and T.M. Plumlee, 2002. Interactive electronic textile development: A review of technologies. J. Textile Apparel Technol. Manage., 2: 1-11.

Natsuki, T., K. Tantrakarn and M. Endo, 2004. Prediction of elastic properties for single-walled carbon nanotube. Carbon, 42: 39-45. DOI: 10.1016/j.carbon.2003.09.011

Odegard, G.M., T.S. Gates, K.E. Wise and L.M. Nicholson, 2002. Equivalent- continuum modeling of nano-structured materials. Composite Sci. Technol., 62: 1869-1880. DOI: 10.1016/S02663538(02)00113-6
Odegard, G.M., T.S. Gates, K.E. Wise, C. Park and E.J. Siochi, 2003. Constitutive modeling of nanotubereinforced polymer composites. Composite Sci. Technol., 63: 1671-1687. DOI: 10.1016/S02663538(03)00063-0

Partridge, A.C., M.L. Jansen and W.M. Arnold, 2000. Conducting polymer based sensors. Materials Sci. Eng. C, 12: 37-42. DOI: 10.1016/S09284931(00)00155-7

Pietraszkiewicz, W., 1984. Lagrangian description and incremental formulation in the non-linear of thin shells. Int. J. Non-Linear Mechanics, 19: 115-140. DOI: 10.1016/0020-7462(84)90002-7

Quin, D., E.C. Dickey, R. Andrews and T. Rantall, 2000. Load transfer and de formation mechanisms in carbon nanotube-polystyrene composites. Applied Physics Lett., 76: 2868-2870. DOI: 10.1063/1.126500

Reissner, E., 1985. Reflections on the theory of elastic plates. Applied Mechan. Rev., 38: 1453-1464. DOI: 10.1115/1.3143699

Rubin, M.B., 2000. Cosserat theories: Shells, rods and points, solid mechanics and its applications series. Kluwer, Dordrecht. DOI: 10.1007/978-94-0159379-3

Sansour, C. and J. Bocko, 1998. On hybrid stress, hybrid strain and enhanced strain finite element formulations for a geometrically exact shell theory with drilling degrees of freedom. Int. J. Numerical Meth. Eng., 435: 175-192. DOI: 10.1002/(SICI)10970207(19980915)43:1<175::AID-

NME448>3.3.CO;2-0

SAP, 2000. A finite element software.

Selmi, A., C. Friebel, I. Doghri and H. Hassis, 2007. Prediction of the elastic properties of single walled carbon nanotube reinforced polymers: A comparative study of several micromechanical models. Composites Sci. Technol., 67: 2071-2084. DOI: 10.1016/j.compscitech.2006.11.016

Varthamanan, Y., 2013. Comparative analysis of reversible logic based carbon nanotube field effect transistormultiplexer performance. Am. J. Applied Sci., 10: 1473-1479. DOI: 10.3844/ajassp.2013.1473.1479 